\title{
Was brauchen wir in ländlichen Räumen? Erreichbarkeitsmodellierung als strategischer Ansatz der regionalen Standort- und Verkehrsplanung
}

\author{
Florian Ahlmeyer ${ }^{1}$ (D) $\cdot$ Dirk Wittowsky $^{1}$ \\ Eingegangen: 7. März 2018 / Angenommen: 15. September 2018 / Online publiziert: 5. November 2018 \\ ๑) Springer-Verlag GmbH Deutschland, ein Teil von Springer Nature 2018
}

\section{Zusammenfassung}

Die Sicherung von Erreichbarkeiten ist ein zentraler Aspekt der Daseinsvorsorge und stellt ländliche Kommunen zunehmend vor große Herausforderungen. Eine zentrale planerische Aufgabe besteht darin, aktuelle und zukünftige Erreichbarkeitssituationen kleinräumig im regionalen Kontext zu erfassen und Räume mit Handlungsbedarf zu identifizieren. Vor allem um die Auswirkungen veränderter Standorte und Verkehrsangebote auf die Erreichbarkeiten abzuschätzen, fehlen bislang geeignete Planungsinstrumente. Für den Landkreis Calw wurde daher im Auftrag des Ministeriums für ländlichen Raum und Verbraucherschutz des Landes Baden-Württemberg ein solches Instrument erarbeitet. Neben der Operationalisierung einer regionalen Erreichbarkeitsanalyse wurde auf Basis lokaler Mobilitätsverhaltensdaten ein Index für die praxisnahe Verwendung entwickelt, der die Erreichbarkeit zu unterschiedlichen Einrichtungen der Daseinsvorsorge zu einem Gesamtindikator zusammenführt und visualisiert. Durch die modellierte Schließung oder Hinzunahme von Einrichtungen bzw. der Veränderung der Reisezeiten können in Szenarien Kennzahlen zur Erreichbarkeit berechnet werden und dadurch als objektive Entscheidungsgrundlage dienen. Dies kann einen wichtigen Beitrag im Abwägungsprozess zur strategischen Daseinsvorsorge leisten. Insbesondere der Erreichbarkeitsindex, der in das hier vorgestellte modular aufgebaute Planungsinstrument zur Erreichbarkeitsmodellierung integriert ist, stellt einen neuen methodischen Ansatz in der Raum- und Standortplanung dar.

Schlüsselwörter Erreichbarkeit $\cdot$ Ländliche Räume $\cdot$ Daseinsvorsorge $\cdot$ Verkehrsmodellierung

\section{What do we need in Rural Areas? How to use Accessibility Modelling as a Strategy in Transport Planning}

\begin{abstract}
Ensuring accessibility to public services and facilities is one of the biggest challenges for rural communities. Thus, planners need to locate spaces with problems in accessibility. It is necessary to estimate the impact of shifts in supply of social infrastructure or public transport. In this particular case, instruments are missing to visualize those shifts. Consequently, such an instrument was developed on behalf of the Ministry of Rural Affairs and Consumer Protection of the federal state of Baden-Württemberg (Germany) in the rural district of Calw. Along with using tools to operationalize a regional analysis of accessibility, an indexing model was developed, which combines in one indicator the accessibilities of public services and facilities providing everyday commodities. Furthermore, the instrument allows removing or adding facilities. It is thus possible to calculate operating figures, which can be used for objective decisions. Valuable for planners developing strategies for organizing services of public interest, the indexing model as part of the developed instrument describes a new method in spatial planning.
\end{abstract}

Keywords Accessibility $\cdot$ Rural space $\cdot$ Public services $\cdot$ Transport modeling

Florian Ahlmeyer

florian.ahlmeyer@ils-forschung.de

Dirk Wittowsky

dirk.wittowsky@ils-forschung.de
1 Forschungsgruppe Alltagsmobilität und Verkehrssysteme, ILS - Institut für Landes- und Stadtentwicklungsforschung, Brüderweg 22-24, 44135 Dortmund, Deutschland 


\section{Regionale Daseinsvorsorge als Planungsziel}

Die ländlichen Regionen in Baden-Württemberg sind geprägt von geringer Bevölkerungsdichte mit dispersen Siedlungsstrukturen. Parallele Prozesse von Wachstum und Schrumpfung schaffen sehr unterschiedliche Bedingungen für die Daseinsvorsorge, sodass existentielle Fragen der Grundversorgung neu diskutiert werden müssen. Aufgrund der demographischen und gesellschaftlichen Veränderungen sowie ökologischen und ökonomischen Herausforderungen werden sich die ländlichen Regionen in den nächsten Jahrzehnten merklich wandeln, sodass eine aktive Anpassungsplanung und Steuerung der Standort- und Verkehrsentwicklung erforderlich werden, um den Menschen Perspektiven und gut erreichbare Standorte und Infrastrukturen der Daseinsvorsorge zu bieten. Die Gewährleistung der gesellschaftlichen Teilhabe durch ein Mindestmaß (Qualität und Quantität) an Infrastrukturversorgung der Daseinsvorsorge sowie die Befriedigung der Mobilitätsbedürfnisse aller Bevölkerungsgruppen gilt als Voraussetzung für die Zukunftsfähigkeit ländlicher Räume.

Doch bereits heute ist das Standortnetz von öffentlichen und privaten Dienstleistungen weitmaschiger als in urbanen Regionen und lange Entfernungen zwischen Wohnund Arbeitsstandorten sowie zu Lebensmittelgeschäften und (Arzt-)Praxen sind die Folge. Aus wirtschaftlichen Gründen ist die Ausdünnung von Standorten der Daseinsvorsorge kaum vermeidbar und führt unweigerlich zu einer Verschlechterung der Erreichbarkeiten, die jedoch erhebliche Konflikte nach sich ziehen kann (BMVI 2015a). Wenn sich dazu parallel der öffentliche Verkehr aus der Fläche zurückzieht, erhöhen sich die negativen Effekte sowohl für die Bevölkerung, indem sie bestimmte Einrichtungen nur noch mit dem Pkw erreichen kann, als auch für die Umwelt, da das individuelle Verkehrsaufkommen steigt. Überlagert werden diese Zukunftsszenarien durch Unsicherheiten im Planungsprozess, sodass neue Planungsansätze zur Transformation bestehender Infrastrukturen sowie regionalspezifische Strategien in der integrierten Raum- und Mobilitätsplanung erforderlich werden. Vor allem die (nahräumliche) Standortattraktivität von Wohnund Gewerbestandorten sind Schlüsselfaktoren, um das Entwicklungspotenzial einer Region in Zeiten heterogener Raumentwicklungen abzuschätzen und resiliente Anpassungsstrategien zu entwerfen (vgl. ARL 2016; BMVI 2016).

Ein nachhaltiges und funktionierendes Mobilitätssystem muss die Erreichbarkeit zu Standorten der Daseinsvorsorge in angemessener Weise garantieren - transaktionskostenarm über einzelne Verkehrsmittel hinaus. Vernetzte Systeme und multimodale Verhaltensweisen werden an Bedeutung gewinnen und damit auch die Entwicklung geeigne- ter kleinräumiger kombinierter Kennwerte zur Beschreibung einer gesamtheitlichen Mobilitätsqualität. Vor allem in ländlichen Räumen, die von Bevölkerungsrückgang betroffen sind, verringert sich die Tragfähigkeit von Infrastrukturleistungen und die des öffentlichen Verkehrs immens. Pilotprojekte versuchen, den Erreichbarkeitsdefiziten durch die Erprobung neuer Mobilitätsformen zu begegnen. Es werden flexible Bedienformen, z. B. in den öffentlichen Verkehr integrierte Mitfahrsysteme oder der Aufbau von Bürgerbussen, gefördert. Das Forschungsvorhaben „Integrierte Mobilitätskonzepte in ländlichen Räumen bei unterschiedlichen Organisationsformen überörtlicher Zusammenarbeit" des Bundesministeriums für Verkehr und digitale Infrastruktur (BMVI) erarbeitete mit Akteuren vor Ort zielgruppenspezifische, vernetzte und integrierte Mobilitätskonzepte (vgl. BMVBS/BBSR 2009; BMVI 2015a; BMVI 2016).

Erreichbarkeitsdefizite ländlicher Räume werden in der übergeordneten Planung zumeist nur angerissen und ohne übergreifende Beschreibung der Daseinsvorsorgequalität erfasst, wie beispielsweise dem aktuellen Raumordnungsbericht des BBSR entnommen werden kann (vgl. BBSR 2017). Das Thema Daseinsvorsorge spielt allenfalls in den ostdeutschen Landesraumordnungsplänen eine zentrale Rolle (BBSR 2017: 29). Auch aktuelle Abhandlungen zu Daseinsvorsorge und Erreichbarkeit behandeln immer nur Teilaspekte wie die hausärztliche Versorgung oder die Notfallinfrastruktur (z. B. Rauch/Rauh 2016; Neumeier 2017). Ländliche Gemeinden und Kreise verfügen zudem kaum über die nötigen Ressourcen, um eine regionale Standort- und Erreichbarkeitsplanung (toolgestützt) eigenständig durchzuführen. Darüber hinaus fehlen oftmals valide Daten, die übergreifend Kategorien der Daseinsvorsorge berücksichtigen, wie auch geeignete praxisnahe Indikatoren, um unterschiedliche Anpassungsstrategien ausarbeiten und objektiv bewerten zu können. Daraus ergibt sich die anwendungsorientierte Forschungsfrage: Wie kann algorithmisch ein Indikatorensystem als regionale Planungsgrundlage entwickelt werden, um Erreichbarkeitsund Versorgungsdefizite verkehrsmittelübergreifend sichtbar und bilanzierbar zu machen?

In dem Forschungsprojekt „Erreichbarkeitssicherung im ländlichen Raum“"wurde ein methodischer Ansatz für eine strategische Daseinsvorsorgeplanung erarbeitet, mit dem Änderungen an der Siedlungs- und Standortstruktur oder am Verkehrsangebot bezüglich ihrer Wirkungen auf die Erreichbarkeit bewertet werden können. Das im Rahmen des Projektes zusammen mit dem Institut für Straßen- und Verkehrswesen der Universität Stuttgart entwickelte integrierte Raum- und Verkehrsplanungstool wurde vom Ministerium für ländlichen Raum und Verbraucherschutz des Landes Baden-Württemberg finanziert und im Landkreis Calw, der als Modellregion ausgewählt wurde, operationalisiert. So- 
wohl die Bevölkerungsentwicklung wie auch die Erreichbarkeit von Einrichtungen der Daseinsvorsorge können für heute und für die Zukunft kleinräumig abgebildet und bewertet werden. Neben der Abbildung klassischer Indikatoren, wie der objektiven Reisezeiten zu einer Kategorie der Daseinsvorsorge ${ }^{1}$, wurde ein multidimensionaler Index determiniert, der Reisezeiten zu Standorten der Daseinsvorsorge gewichtet, bilanziert und als ,Früherkennungsindikator' für strategische Planungen eingesetzt werden kann.

Mit dem integrierten Raum- und Verkehrsplanungswerkzeug (tool) werden vielfältige Bewertungen und Abschätzungen von infrastrukturellen Maßnahmen und objektive Aussagen über Reisezeiten zu Standorten der Daseinsvorsorge mit unterschiedlichen Verkehrsmitteln wie dem motorisierten Individualverkehr, öffentlichen Personenverkehr, zu Fuß und mit dem Fahrrad ermöglicht. Allein die erwarteten Konsequenzen, wie der Rückgang wirtschaftlicher Aktivität oder die Schließung von Einrichtungen der Daseinsvorsorge, die aus demographischen Verschiebungen und knappen Finanzbudgets der öffentlichen Hand resultieren, üben weiteren Druck auf die Raum- und Mobilitätsplanung aus. Für die Standortplanung der öffentlichen Hand ergibt sich ein Dilemma: Einrichtungen müssen aus wirtschaftlichen Gründen geschlossen werden, wodurch sich die Erreichbarkeitsbedingungen verschlechtern. So können negative Anreize für eine Abwanderung entstehen, welche eine Abwärtsspirale in Gang setzen, die eine Aufrechterhaltung eines Mindestangebots an Einrichtungen und Infrastrukturen bedroht und damit auch den Zusammenhalt von Gesellschaften in ländlichen Räumen neu konstituiert. Hier muss die Planung aufzeigen, wie Defizite auch mit geringerem Budget beseitigt werden können. Durch den Erreichbarkeitsindex können somit objektiv Räume identifiziert werden, wo im Landkreis Calw ein Versorgungs- und Mobilitätsdefizit entsteht. Gezielte Strategien können anschließend die Attraktivität der betroffenen Regionen erhalten.

Mit dem vorliegenden Beitrag sollen insbesondere die Entwicklung (Kapitel 4) und die Anwendungsmöglichkeiten (Kapitel 5) des Indikatorensystems aufgezeigt werden, welches die Erreichbarkeit zu den unterschiedlichen Einrichtungen der Daseinsvorsorge in seiner Gesamtheit betrachtet. Zuvor wird in Kapitel 2 der Forschungsstand zu Erreichbarkeit aufgearbeitet, bevor in Kapitel 3 der Aufbau des Modells erläutert wird. Kapitel 6 zählt Anwendungsmöglichkeiten in der Fachplanung auf.

\footnotetext{
1 Vgl. z. B. Erreichbarkeitsatlas Metropolregion Hamburg (http:// geoportal.metropolregion.hamburg.de (12.07.2018)) und Erreichbarkeitsatlas für die Europäische Metropolregion München (https:// www.sv.bgu.tum.de/forschung-und-beratung/projekte/projekte/einerreichbarkeitsatlas-fuer-die-europaeische-metropolregion-muenchen$\mathrm{emm} /(12.07 .2018))$.
}

\section{Grundlagen der Erreichbarkeit}

Der demographische Wandel mit seinen Auswirkungen auf ländliche Räume und das Verkehrsverhalten werden bereits seit mehr als zehn Jahren in der geographischen Forschung sowie der Mobilitätsforschung diskutiert (vgl. z. B. Kemper 2004; Kilper/Müller 2005; FGSV 2006; Ottmann 2010). Detaillierte Erreichbarkeitsuntersuchungen standen jedoch zu jener Zeit nicht auf der Agenda. Für den öffentlichen Verkehr entstand zwar eine Fülle von Strategien wie z. B. Planungen zu flexiblen Bedienformen (Wittowsky 2008; BMVBS/BBSR 2009) oder einer prinzipiellen Subjektförderung zur Mobilitätssicherung, wie der Ausgabe von Taxischeinen an mobilitätseingeschränkte Personen oder an Haushalte mit Schülern (Canzler/Karl 2010). Diese basieren aber nicht auf Analysen zur Erreichbarkeit, sondern stellen Konzepte zur Veränderung des Verkehrssystems dar. Auch die Zusammenhänge und Konsequenzen zur Daseinsvorsorge, wie der zunehmende Rückzug von Einrichtungen aus der Fläche, sind bekannt (vgl. z. B. BMVBS/BBSR 2011; Proske 2011).

Erreichbarkeiten spielen in der Mobilitätsforschung eine zentrale Rolle, werden jedoch unterschiedlich definiert und operationalisiert. Nach Geurs und van Wee (2004) liegt der Erreichbarkeit ein Wirkungsgefüge zugrunde, dessen Komponenten aus der Landnutzung, dem Verkehrssystem, den zeitlichen Ressourcen sowie den individuellen Ressourcen einer jeweiligen Person bestehen. Gather, Kagermeier und Lanzendorf (2008) hingegen unterscheiden zwischen personenbezogener Erreichbarkeit, also den Gelegenheiten, die durch ein bestimmtes Verkehrsmittel in der entsprechenden Wegzeit erreicht werden können (access), und den Einzugsbereichen, die beschreiben, wie viele Personen erreicht werden können (accessibility). Im vorliegenden Beitrag wird Erreichbarkeit personenbezogen betrachtet und somit ausgehend von den Nachfragern gemessen. Da nicht jeder Nachfrager einzeln erfasst oder modelliert wird, werden diese in Rasterzellen mit den entsprechenden Bevölkerungsangaben zusammengefasst (vgl. auch Kapitel 3).

Die Erreichbarkeitsverhältnisse als solche zu erforschen, um Instrumente mit einer höheren Übertragbarkeit auf andere Räume entwickeln zu können, rückt erst in jüngster Vergangenheit zunehmend in den Fokus (vgl. Neumeier 2013; Rauch/Rauh 2016). Erreichbarkeiten werden auf der deutschlandweiten Ebene häufig nur mittels einfacher Analysen dargestellt, da sonst eine zu hohe Komplexität entsteht. So untersucht das Thünen-Institut für ländliche Räume lediglich die Erreichbarkeit einzelner Einrichtungen der Daseinsvorsorge wie Apotheken, Supermärkte oder Hausärzte (Neumeier 2013; Neumeier 2014; Neumeier 2017). Das Bundesinstitut für Bau-, Stadt- und Raumforschung 
nutzt als Kennwert Luftliniendistanzen ${ }^{2}$, die nur sehr grob die tatsächlichen Erreichbarkeitsverhältnisse abbilden. In Bezug auf Erreichbarkeitsindikatoren machen Bertolini, Le Clercq und Capoen (2005) deutlich, dass immer ein gewisses Spannungsfeld zwischen komplexen Indikatoren und der einfachen Nachvollziehbarkeit besteht. So sind einfache Indikatoren, wie die Reisezeit zum nächsten Arzt, leicht nachvollziehbar, zeigen aber nur einen Teilaspekt der Erreichbarkeit auf. Komplexere Indikatorensysteme, die auf einer Widerstandsfunktion beruhen, mehrere Ziele miteinander kombinieren und normalisierte Werte abbilden, haben einen höheren Informationsgehalt, sind jedoch nicht auf Anhieb nachvollziehbar. Auch andere Autoren heben dies in Bezug auf den Planungsprozess hervor (vgl. Halden 2002; Geurs/van Wee 2004; Wulfhorst/Büttner/Ji 2017). Nachfolgend werden die methodentheoretischen Grundlagen vorgestellt, die der Entwicklung des neuen Erreichbarkeitsindexes zugrunde liegen.

Modelle für kombinierte Erreichbarkeitsindizes (z. B. Walk Score, walkability) werden vornehmlich für die fußläufige Erreichbarkeit aufgebaut (vgl. Frank/Sallis/Saelens et al. 2010; Walk Score 2011; Dinkel 2014). Generell bezieht sich die Erreichbarkeitsdebatte vor allem auf städtische Räume (Rode 2014). Erreichbarkeitsindizes können aber gerade in ländlichen Räumen als Schlüsselindikatoren dienen, um die Versorgungsdichte evaluieren zu können. Bei Walkability-Ansätzen wird die nahräumliche Erreichbarkeit darüber definiert, wie fußgängerfreundlich die Umgebung anhand von Raumstrukturdaten einzuschätzen ist. Dabei werden Kennwerte wie Siedlungs- und Einzelhandelsdichte, Konnektivität und der Gebäudefunktionsmix verwendet (vgl. Frank/Sallis/Saelens et al. 2010). Somit modellieren diese keine echten Erreichbarkeiten, da nicht die Weglängen zu den relevanten Zielen gemessen werden. Der Einsatz des Index ist in ländlichen Räumen jedoch nicht sinnvoll, da von aggregierten Werten, wie Siedlungsund Einzelhandelsdichte, nicht auf die Dichte nahräumlicher Versorgungseinrichtungen geschlossen werden kann. Das Grundprinzip des Walk Score eignet sich eher, da dieser mit Distanzwerten als Basiskennwert operiert. Der Walk Score besteht in der Anwendung einer Widerstandsfunktion zur Normalisierung der Reisezeiten auf einer Skala von 0 bis 100. Doch bei den Zielen, die jener Index verwendet, steht nicht die Grunddaseinsvorsorge im Vordergrund, sondern vielmehr der Einfluss wohnortnaher, fußläufiger Erreichbarkeit auf die Immobilienpreise in urbanen Räumen (vgl. Walk Score 2011). Als sehr komplexes Beispiel ist an dieser Stelle das Land Use and Public Transport Accessibility Indexing Model (LUPTAI) aufzuführen, das die Erschließung der Haltestellen, die Reisezeiten im öffent-

\footnotetext{
2 https://www.bbsr.bund.de/BBSR/DE/Home/Topthemen/nahversor
} gung_land.html (15.07.2018) lichen Verkehr und den Zugang zu unterschiedlichen Zielen der Daseinsvorsorge miteinander kombiniert (vgl. Yigitcanlar/Sipe/Evans et al. 2007). Die hier aufgeführten theoretischen Erkenntnisse dienen primär als Grundlage für die Entwicklung eines optimierten Erreichbarkeitsindexes, der als Kennwert in das Modell integriert wird.

In Bezug auf die Entwicklung komplexer Indikatoren zur Bewertung der Erreichbarkeit zu den Zielen der Daseinsvorsorge besteht eine Forschungslücke, da es noch keinen kombinierten Kennwert zur Beurteilung der regionalen Daseinsvorsorge gibt. Dies wird mit dem Aufbau des in Kapitel 4 vorgestellten Erreichbarkeitsindexes erprobt, indem alle Einrichtungen der Daseinsvorsorge kleinteilig in einen modular aufgebauten Index mit eingebunden werden. Zuvor wird in Kapitel 3 das zugrunde liegende Modell beschrieben.

\section{Modellentwicklung}

Der räumliche Umgriff des Untersuchungsraumes erstreckt sich auf das Gebiet des Landkreises Calw mit 155.414 Einwohnern im Jahr 2015 und einer Fläche von 797,15 $\mathrm{km}^{2}{ }^{3}$ Das Untersuchungsgebiet repräsentiert unterschiedliche Gegebenheiten in ländlichen Räumen und ist somit als Modellraum zur Analyse von Erreichbarkeiten gut geeignet. So finden sich verdichtete Gebiete mit den Städten Calw und Nagold, flächenhaft strukturierte, dünner besiedelte Gebiete mit umliegenden Dörfern im zentralen Bereich des Kreises und, begründet durch die Lage im Randbereich des Schwarzwaldes, bandartige Siedlungsanordnungen in Tallage im westlichen Bereich des Landkreises.

Für das Modell wird ein Erreichbarkeitskonzept verfolgt, das die Erreichbarkeit von der Position der Nachfrager aus bemisst. Um jedoch anwendbare und planerisch verwertbare Ergebnisse zu erzielen, wird nicht jedes Individuum berücksichtigt, sondern die Einwohner werden in Rasterzellen mit einer Kantenlänge von 200 Metern zusammengefasst. Die Analysen sind kleinräumiger als bei Ansätzen, die sich auf Gemeindegrenzen beziehen, betrachten jedoch nicht die Ebene des Individuums, was im Modell kaum operationalisierbar wäre. Da im Modell die Erreichbarkeit zu Zielen der Daseinsvorsorge dargestellt wird, sind die Reisezeiten ausgehend von den Mittelpunkten der Rasterzellen zu den jeweils nächsten Zielen einer Einrichtungskategorie (z. B. nächster Supermarkt oder nächste Grundschule; vgl. auch Abbildung 1) von zentraler Bedeutung. Ziel ist es, ein Planungsinstrument zu installieren, welches den Kreisen in Zusammenarbeit mit den Gemeinden ermöglicht, die Qualität der Daseinsvorsorge in Bezug auf die Erreichbarkeit bewerten zu können und mögliche Auswirkungen von

\footnotetext{
${ }^{3}$ Die Daten wurden vom Landkreis Calw zur Verfügung gestellt.
} 
Abb. 1 Gewichtungsfaktoren im Erreichbarkeitsindex

\begin{tabular}{|c|c|c|c|c|}
\hline $\begin{array}{l}\text { Einrichtungs- } \\
\text { kategorie }\end{array}$ & $\begin{array}{c}\text { Bereiche der } \\
\text { Daseinsvorsorge }\end{array}$ & Gewicht & \multicolumn{2}{|c|}{ Erläuterung Schritte } \\
\hline Grundschule & \multirow{3}{*}{$\begin{array}{l}\text { Bildung } \\
(15 \%)\end{array}$} & 5 & \multirow{6}{*}{ Schritt 1} & \\
\hline Sekundarstufe II & & 5 & & Wegzweck- \\
\hline Kitas & & 5 & & vertellung \\
\hline Hausarzt & \multirow{5}{*}{$\begin{array}{l}\text { Gesundheit } \\
\text { (25\%) }\end{array}$} & 11 & & $\begin{array}{l}\text { gemals der } \\
\text { Haushalts- }\end{array}$ \\
\hline Fachärzte & & 6 & & hausnalts- \\
\hline Krankenhaus & & 3 & & \\
\hline Apotheke & & 3 & & \\
\hline Pflegeheim & & 2 & & \\
\hline Bäcker & \multirow{6}{*}{$\begin{array}{c}\text { Versorgung } \\
\text { (30\%) }\end{array}$} & 4,5 & & \\
\hline Metzger & & 4,5 & \multirow{3}{*}{ Schritt 2} & \multirow{3}{*}{$\begin{array}{c}\text { Einbezug lokaler } \\
\text { Akteure }\end{array}$} \\
\hline Supermarkt & & 9 & & \\
\hline Lebensmittel & & 6 & & \\
\hline Drogerie & & 3 & & \\
\hline Tankstelle & & 3 & & \\
\hline Bücherei & \multirow{7}{*}{$\begin{array}{l}\text { Freizeit und Kultur } \\
\qquad(20 \%)\end{array}$} & 1 & & \\
\hline Kino & & 2 & \multirow{3}{*}{ Schritt 3} & \multirow{3}{*}{$\begin{array}{l}\text { Einbezug } \\
\text { theoretischer } \\
\text { Erkenntnisse }\end{array}$} \\
\hline Theater & & 1 & & \\
\hline Kirche & & 3 & & \\
\hline Sportplatz & & 4 & & \\
\hline Schwimmbad & & 3 & & \\
\hline Restaurant & & 6 & & \\
\hline Bank & \multirow{6}{*}{$\begin{array}{l}\text { Dienstleistungen und Behörden } \\
\qquad(10 \%)\end{array}$} & 2 & \multirow{6}{*}{ Schritt 4} & \multirow{6}{*}{$\begin{array}{l}\text { Anpassungen zur } \\
\text { besseren } \\
\text { Operationali- } \\
\text { sierbarkeit im } \\
\text { Modell }\end{array}$} \\
\hline Post & & 2 & & \\
\hline Frisör & & 2 & & \\
\hline Optiker & & 1 & & \\
\hline Amt & & 2 & & \\
\hline Jobcenter & & 1 & & \\
\hline \multicolumn{2}{|r|}{ Summe } & 100 & & \\
\hline
\end{tabular}

veränderten Standort- und Angebotskonzepten abzuschätzen. Um dies anwendungsorientiert zu gewährleisten, wurde ein Erreichbarkeitsindex entwickelt und in das Modell integriert.

Das Planungswerkzeug besteht aus fünf Modulen, die eng miteinander verzahnt sind (vgl. Abbildung 2). Dem Bevölkerungsmodell liegen Bevölkerungsdaten auf Ortsteilebene sowie Einwohnerprognosedaten für das Jahr 2030 auf Gemeindeebene zugrunde. Für das Bevölkerungsmodell werden die Einwohner auf Grundlage des Projekts GEOSTAT $^{4}$ und dem Anteil der Siedlungsfläche des IÖRMonitors für Siedlungs- und Freiraumentwicklung ${ }^{5}$ auf Rasterzellen mit einer Kantenlänge von 200 Metern disaggregiert. Die GEOSTAT-Daten beinhalten Einwohnerdaten auf einer Kantenlänge von 1.000 Metern. Diese wurden mit IÖR-Daten, die den Anteil der Siedlungsfläche an der Gesamtfläche mit der Kantenlänge von 100 Metern beinhalten, verrechnet und somit die Einwohnerzahl modelliert.

\footnotetext{
${ }^{4} \mathrm{http} / / / \mathrm{ec}$. europa.eu/eurostat/documents/4311134/4350174/ESSnetproject-GEOSTAT1A-final-report_0.pdf/fc048569-bc1c-4d99-95970ea0716efac3 (16.07.2018).

5 http://www.ioer-monitor.de (16.07.2018) des Leibniz-Instituts für ökologische Raumentwicklung.
}

Anschließend wurden je vier Zellen zusammengefasst, um die entsprechende Auflösung zu erreichen. Es werden nur solche Gebiete betrachtet, die auch besiedelt sind. Hieraus resultieren 4.419 Rasterzellen, für die kombiniert mit 5.252 Zielen 23.208.588 Relationen Reisezeiten und Entfernungen zu berechnen sind. Die Kantenlänge von 200 Metern wurde gewählt, da sie eine detaillierte Darstellung ermöglicht. Die Anzahl der Relationen bleibt jedoch handhabbar.

Insgesamt wurden 5.252 Standorte für das Standortmodell lokalisiert, wobei sich 2.426 Standorte innerhalb und 2.862 außerhalb des Landkreises Calw befinden. Die im Modell ermittelten und georeferenzierten Standorte setzen sich aus unterschiedlichen Quellen zusammen (unter anderem kommunale Daten, Open-Street-Map, Deutsche Post). Hierfür wurden definierte Kategorien gebildet, die aus den klassischen Bereichen der Daseinsvorsorge bestehen (vgl. auch Abbildung 1).

Um die verkehrliche Angebotsqualität und damit die Erreichbarkeit der Einrichtungen mit verschiedenen Verkehrsmitteln (Fuß, Rad, Pkw und öffentlicher Verkehr (ÖV)) zu bestimmen, wird ein vereinfachtes Verkehrsmodell aufgebaut, welches das Verkehrsangebot und die Verkehrsnachfrage beinhaltet. Das Verkehrsmodell kann für den heutigen Zustand und für künftige Zustände die Erreichbarkeit 
Abb. 2 Module des Planungswerkzeugs

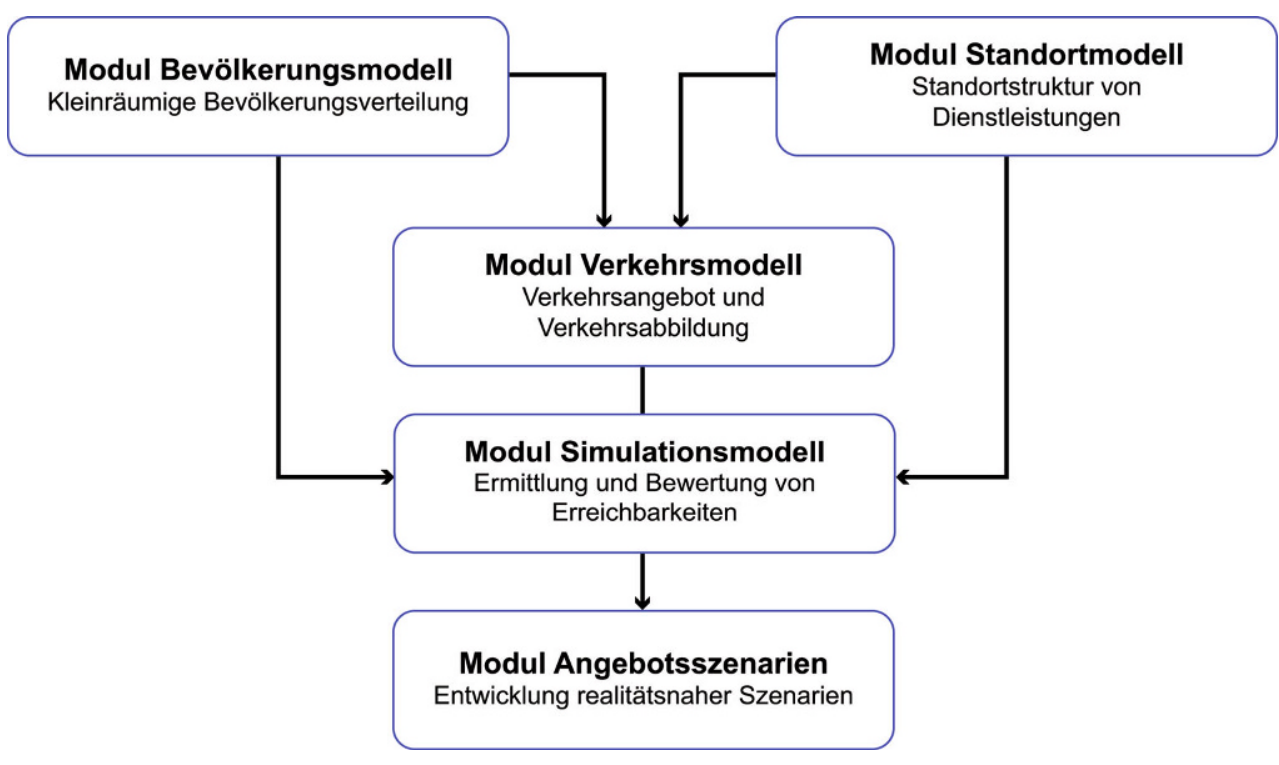

mit verschiedenen Kenngrößen wie Reisezeit, Bedienungsoder Umstiegshäufigkeit quantifizieren und bewerten. Die Grundlage für das Netzmodell, welches über die Verkehrsplanungssoftware VISUM operationalisiert wird, bildet die frei zugängliche Geodatenbank Open-Street-Map (OSM).

Der Individualverkehr (Fuß-, Rad- und Pkw-Verkehr) orientiert sich am Straßennetz von OSM. Für die Abbildung der Erreichbarkeit des öffentlichen Verkehrs dienen die importierten Fahrplandaten der Nahverkehrsgesellschaft Baden-Württemberg (NVBW). Die Fahrzeit wird von den Zellenschwerpunkten der Quellraster hin zu den Zellenschwerpunkten der Zielraster ermittelt. Dabei wird der Anschluss der Bezirke (Raster) an das Streckennetz durch Anbindungen als $\mathrm{Zu}$ - und Abgangsweg mitberücksichtigt. Neumeier (2014: 24f.) stellt beim Vergleich unterschiedlicher Studien fest, dass die normale Gehgeschwindigkeit bei mindestens 4,7 km/h liegt. Da nicht davon ausgegangen werden kann, dass Fußwege ohne Verlustzeiten, die durch Wartezeiten bei der Querung von Straßen oder Lichtsignalanlagen entstehen, durchgeführt werden können, wird für den Fußverkehr durchgängig eine Geschwindigkeit von $4 \mathrm{~km} / \mathrm{h}$ angenommen. Die Geschwindigkeitsermittlung für den Radverkehr berücksichtigt Höhenunterschiede und erfolgt nach Gölz (2007). Die Ermittlung der Fahrtzeiten des Pkw-Verkehrs basiert auf den zulässigen Höchstgeschwindigkeiten auf den jeweiligen Straßenabschnitten. An den Knotenpunkten mit Lichtsignalanlagen wurde eine Kfz-Verlustzeit von $15 \mathrm{Se}$ kunden und an allen übrigen Knoten von 3 Sekunden angenommen, um eine realistischere Fahrtzeit im unbelasteten Netz zu ermitteln (vgl. Friedrich/Siedentop/Ahlmeyer et al. 2016).

Die Ermittlung der Fahrtzeiten für den öffentlichen Verkehr obliegen einer komplexeren Berechnung. Die Anbindung an das Straßennetz erfolgt nach dem für den Indivi- dualverkehr aufgeführten Prinzip. Die gesamte Reisezeit für den öffentlichen Verkehr wird aus der Summe der Zu- und Abgangszeit (4 km/h), Startwartezeit, Fahrtzeit im Fahrzeug, Umsteigewartezeit und Gehzeit gebildet. Das Modell berechnet auf der Basis der Angebotsqualitäten der unterschiedlichen Verkehrsmodi und den Standorten der Daseinsvorsorge unterschiedliche Kenngrößen der Erreichbarkeit (z. B. Reisezeit zum nächsten Standort, mittlere Reisezeit, Bedienungshäufigkeit, Umstiegshäufigkeit) für jede ausgewählte Relation (vgl. Friedrich/Siedentop/Ahlmeyer et al. 2016).

Als Folge einer veränderten Standortkonfiguration der Standorte der Daseinsvorsorge oder einer Veränderung der Angebotsstruktur im Individual- oder öffentlichen Verkehr bildet das Modell die Erreichbarkeitsveränderungen in der Reisezeit ab. Somit kann das Planungsinstrument die Erreichbarkeiten und mögliche Änderungen der Standortstruktur der Daseinsvorsorge bilanzierbar machen. Auf diese Weise ermöglicht das Modell Szenario-Berechnungen, um beispielsweise offenzulegen, wo eine strukturelle Vulnerabilität besteht oder wo die Erreichbarkeitssituation Anpassungsstrategien erfordert. Die Szenarien können helfen, Folgen möglicher Anpassungsmaßnahmen abzuschätzen und politische Debatten zu versachlichen: Wie wirkt sich die Schließung einer Schule aus? Wie viele Schüler fahren wie viel länger? Wie viel Prozent der Bevölkerung erreichen innerhalb einer halben Stunde den nächsten Arzt? In welcher Zeit kann der nächste Supermarkt erreicht werden? Bestehen Alternativen zur nächstgelegenen Einrichtung der Daseinsvorsorge?

Dem Landkreis Calw steht somit ein Raum- und Verkehrsplanungstool zur Verfügung, mit dem die Folgen sowohl von Maßnahmen als auch von zukünftigen Entwicklungen abgeschätzt werden können. Obwohl es sich um ein 
komplexes Modell handelt, das auf der Basis einer kommerziellen Software erarbeitet wurde, liegen für den Landkreis die Vorteile auf der Hand. So können neben der Erreichbarkeitsanalyse und der Simulation veränderter Standortstrukturen in Echtzeit klassische Aufgaben der Verkehrsplanung durchgeführt werden, wie die integrierte Abbildung des Angebotes des Individual- und öffentlichen Verkehrs, die Berechnung der Verkehrsnachfrage auf der Grundlage von Strukturdaten sowie der Umlegung der Verkehrsnachfrage auf die einzelnen Verkehrssysteme. Zukünftig können somit auch Erreichbarkeitsanalysen mit Berechnungen zur Verkehrsnachfrage verknüpft und integriert analysiert werden. Trotzdem ist kritisch anzumerken, dass gegenüber einer Open-Source-Lösung Nutzungshemmnisse entstehen. Eine Möglichkeit, diese Zugangsbarrieren zu vermindern, stellt die Bereitstellung eines onlinebasierten Präsentationsund Eingabe-Moduls (Frontend) seitens des Landkreises dar, welches es den Gemeinden ermöglicht, nach einer einmaligen Initialberechnung eigenständig Erreichbarkeitsanalysen und Szenarioberechnungen durchzuführen.

\section{Erreichbarkeitsindex}

Für die objektive Entscheidungsunterstützung in der Planungsphase oder auf der strategischen Ebene sind für die Bewertung regionaler Strategien der Daseinsvorsorge integrierte Betrachtungen und aussagekräftige Indikatoren notwendig. Die Güte der Erreichbarkeit wird anhand von Reisezeiten von den Quellorten zu den Einrichtungen der Daseinsvorsorge gemessen. Zur Darstellung der Robustheit eines Wohnstandortes - z. B. für den Fall wegbrechender Standorte der Daseinsvorsorge - wird die Reisezeit zum nächsten und übernächsten Standort betrachtet. Werden jedoch mehrere Standorte der Daseinsvorsorge integriert betrachtet, stellen isolierte Reisezeiten keine ausreichende Basis dar, um die Erreichbarkeit zu den unterschiedlichen Einrichtungsarten insgesamt aufzuzeigen. Dinkel (2014: 20ff.) und Schwarze (2005: $9 \mathrm{ff}$.) fassen in ihren Ausführungen gut zusammen, wie ein solcher Erreichbarkeitsindikator, zu denen auch der bereits aufgeführte Walk Score zählt, theoretisch fundiert generiert werden kann. Diese Ausführungen bilden im Wesentlichen die theoretische Grundlage für den Aufbau der Widerstandsfunktion des Erreichbarkeitsindexes. Der Aufbau der Gewichtung orientiert sich im Wesentlichen an der Methodik, die der Bildung des Walk Score (2011) zugrunde liegt, mit dem Unterschied, dass zusätzlich zu den theoretischen Annahmen auch die empirischen Wegedaten der Haushaltsbefragung mit einfließen.

\subsection{Aufbau des Erreichbarkeitsindexes}

Der Erreichbarkeitsindex basiert auf theoretischen Grundlagen bereits aufgeführter Indizes (Walk Score 2011; Dinkel 2014; Frank/Sallis/Saelens et al. 2010) sowie einer repräsentativen Mobilitätsbefragung im Landkreis Calw aus dem Jahr 2013, die Teil einer landkreisweiten Haushaltsbefragung war. Die Haushaltsbefragung war eine schriftlich-postalische Stichtagsbefragung. Die Gewichtung der Antworten der insgesamt 3.595 Teilnehmer erfolgte nach Geschlecht (gemeindespezifisch), Altersgruppe (kreisweit) und der Hochrechnung nach Einwohnerzahl je Gemeinde (vgl. Friedrich/Siedentop/Ahlmeyer et al. 2016). Wesentlicher Teil der Mobilitätsbefragung ist das Wegetagebuch, in dem insgesamt 11.850 Wege erfasst wurden. Aus den empirischen Daten des Wegetagebuches als zentralem Bestandteil der Haushaltsbefragung wurde eine kumulierte Reisezeit-Widerstandsfunktion ${ }^{6}$ abgeleitet. Diese Funktion basiert auf den erhobenen Wegezeiten, die im Rahmen der Haushaltsbefragung für verschiedene Wegzwecke erhoben wurden. Grundstock sind die Häufigkeiten der Wege ab einem Wert von 10 Minuten, da bis zu diesem Zeitaufwand von einer sehr guten Erreichbarkeit für den Landkreis Calw ausgegangen werden kann (vgl. Kickner 1998). Für die Bildung der Widerstandsfunktion werden nur relevante Wegzwecke und Verkehrsmittel einbezogen, die in Tabelle 1 aufgeführt sind. Insgesamt sind für die Berechnung des Erreichbarkeitsindex vier Wegzwecke und Verkehrsmittel berücksichtigt worden. Diese sind in Tabelle 1 kursiv hervorgehoben.?

Zudem werden nur Wege betrachtet, die von der Wohnung aus starten oder dort enden. Dadurch können Wege ausgeschlossen werden, die nicht für die Daseinsvorsorge relevant sind. Dazu gehören beispielsweise Wege, die von der Arbeit direkt zum Einkaufen führen und möglicherweise viel länger sind als solche, die für die wohnortnahe Versorgung relevant sind. Die Widerstandsfunktion wird aus den kumulierten Wegzeitaufwandsverteilungen differenziert nach den Hauptverkehrsmitteln generiert (vgl. Abbildung 3). Die Grundgesamtheit für die Wege ab $\geq 10$ min beträgt $n=1.532$ für den Pkw, $n=717$ für den öffentlichen Verkehr, $n=498$ für die Fußwege und $n=136$ für die Fahrradwege. Abbildung 3 führt die Funktionen auf, auf deren Grundlage die finale Widerstandsfunktion ermittelt wurde. Die kumulierte Weglängenverteilung nach Verkehrsmitteln, dargestellt durch die farbigen Kurven in Abbil-

\footnotetext{
${ }^{6}$ Die Widerstandsfunktion stellt den zu überwindenden Raumwiderstand dar (vgl. Schwarze 2005: 11), die in dieser Ausarbeitung aus den kumulierten Wegzeitaufwandsverteilungen gebildet wird.

7 SPNV und ÖPNV werden zu öffentlichem Verkehr zusammengefasst, zudem wird im Modell nicht zwischen Selbstfahrern und Mitfahrern beim motorisierten Individualverkehr unterschieden.
} 
Tab. 1 Wegzwecke und Verkehrsmittel in der Haushaltsbefragung des Landkreises Calw

\begin{tabular}{llll}
\hline Wegzwecke & & Verkehrsmittel & $14,8 \%$ \\
\hline Zur Arbeit & $23,1 \%$ & Fuß & $4,3 \%$ \\
Dienstlich /geschäftlich & $6,2 \%$ & Fahrrad & $0,3 \%$ \\
ZurSchule /zur Ausbildung & $10,3 \%$ & Pedelec/E-Bike & $0,7 \%$ \\
Einkaufen (täglicher Bedarf) & $13,7 \%$ & Kraftrad & $59,5 \%$ \\
Einkaufen (längerfristiger Bedarf) & $3,2 \%$ & Pkw Fahrer & $11,6 \%$ \\
Private Erledigungen & $12,3 \%$ & Pkw Mitfahrer & $0,1 \%$ \\
Freizeit & $22,6 \%$ & Taxi & $1,9 \%$ \\
Begleitung von Personen & $7,2 \%$ & SPNV & $6,8 \%$ \\
Sonstiges & $1,4 \%$ & ÖPNV & \\
$\mathrm{n}=11.850$ & & &
\end{tabular}

Verwendete Wegzwecke und Verkehrsmittel sind kursiv gedruckt

ÖPNV: Öffentlicher Personennahverkehr, SPNV: Schienenpersonennahverkehr

Abb. 3 Weg-Zeit-Verteilung nach Verkehrsmitteln und den daraus resultierenden Funktionen

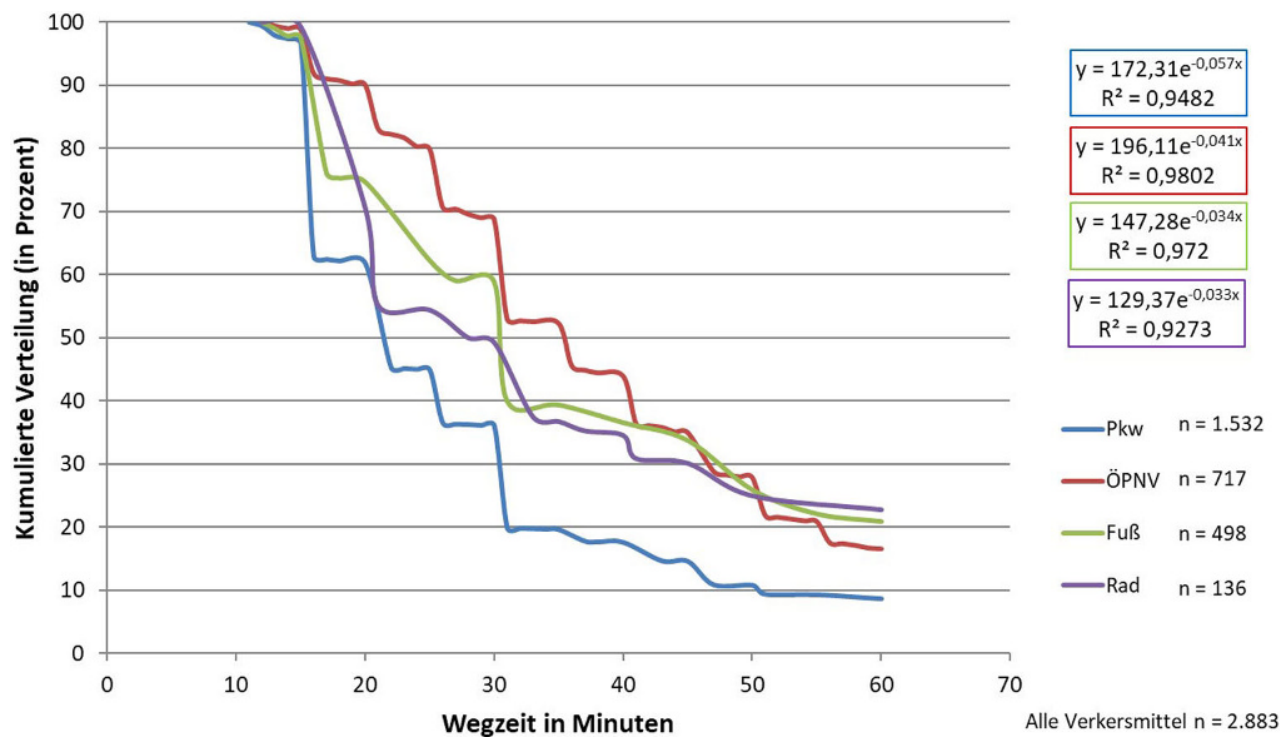

dung 3, bilden die Grundlage für die Berechnungen der einzelnen Exponentialfunktionen $\left(y=172,31 \mathrm{e}^{-0,057 x}\right.$ für den motorisierten Individualverkehr, $y=196,11 \mathrm{e}^{-0,041 \mathrm{x}}$ für den öffentlichen Verkehr, $y=147,28 \mathrm{e}^{-0,034 x}$ für den Fußverkehr und $y=129,37 e^{-0,033 x}$ für den Radverkehr). Die für die vier Funktionen ermittelten Minutenwerte für den Fuß-, Rad-, Pkwund öffentlichen Verkehr wurden mit den Minutenwerten 1 bis 60 verrechnet. Aus diesen idealisierten Werten wurde die finale Widerstandskurve ermittelt. Die 2.883 Wege, die aus der Haushaltsbefragung extrahiert wurden, bilden die empirische Grundlage für die in Formel (1) aufgeführte Widerstandsfunktion $\mathrm{y}=146,11 \mathrm{e}^{-0,039 x}$ (vgl. Abbildung 4). Die Werte, die aus den Funktionen der jeweiligen Hauptverkehrsmittel resultieren, fließen zu je gleichen Anteilen in die finale Widerstandsfunktion ein. Die Formeln bilden hier die Berechnungsgrundlage, um eine Funktion ermitteln zu können, die unabhängig von der Nutzungshäufigkeit alle Verkehrsmittel gleich berücksichtigt, da sonst Werte ermittelt worden wären, die den nicht motorisierten Verkehrsteil- nehmern nicht gerecht würden. Ohne die gleiche Gewichtung der unterschiedlichen Verkehrsmittel wären die Wege des motorisierten Individualverkehrs überrepräsentiert.

Die für das Werkzeug angewandte Widerstandsfunktion unterscheidet sich von einer idealtypischen Funktion dahingehend, dass sie aus lokalen empirischen Daten abgeleitet wurde, wodurch der Erreichbarkeitsindex regionalspezifisch und weniger normativ ist.

Für die Berechnung des Index wird für die Punkteverteilung eine Ober- und Untergrenze festgelegt. Alle aus dem Modell generierten Reisezeiten unter 10 Minuten erlangen 100 Punkte. Zeiten von über 60 Minuten erhalten einen Wert von 0 , da ab einem Zeitaufwand von über 60 Minuten eine geringe Erreichbarkeitsqualität konstatiert werden kann (vgl. Kickner 1998).

Abgeleitet aus den vorausgegangenen Ausführungen gelten Formel (1) und Formel (2) als Berechnungsgrundlage. Der Index kann für den Individualverkehr mithilfe der aufgeführten Berechnungsformel gebildet werden. Für den In- 
Abb. 4 Widerstandskurve mit Funktionsgleichung für den Erreichbarkeitsindex

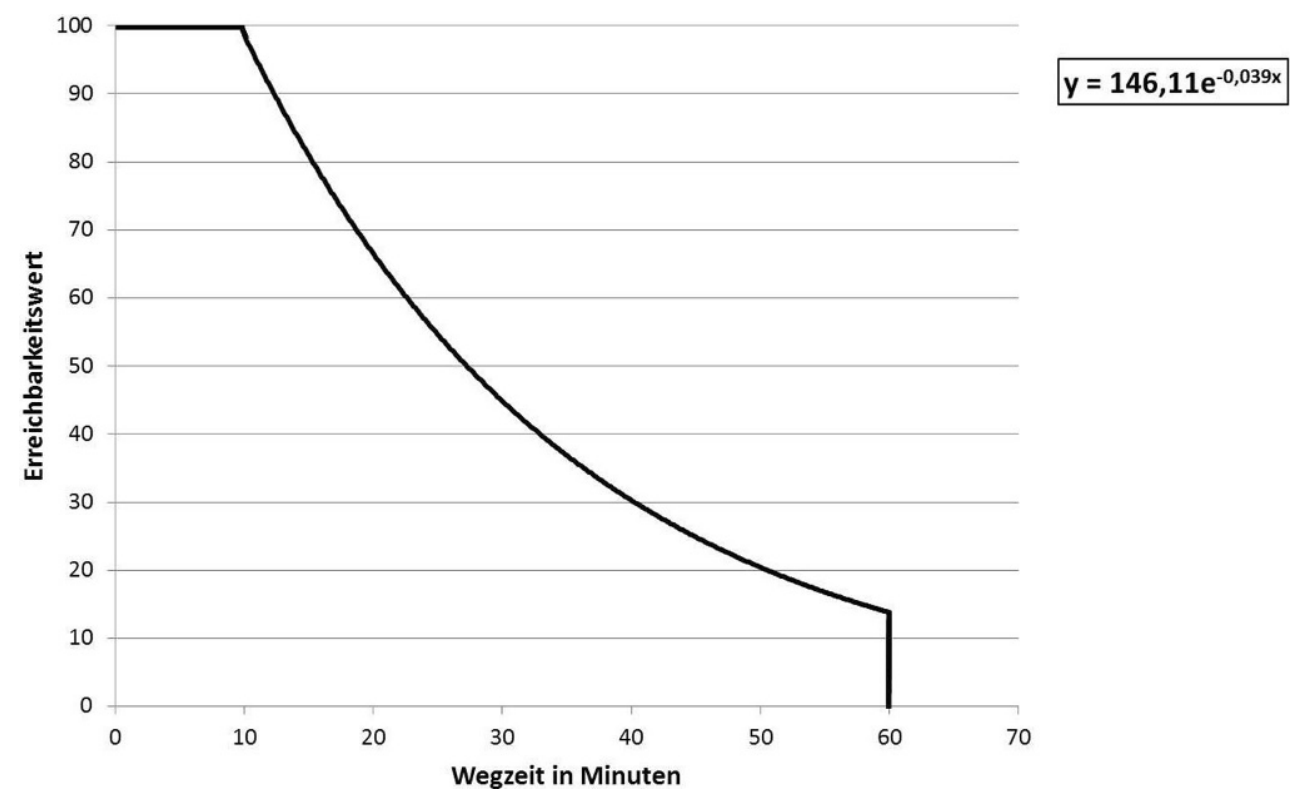

dividualverkehr gelten lediglich die Reisezeiten als Grundlage für den Indexwert.

$$
E(\text { Index })=\sum_{j} G(Z j) * f_{i j}\left(146,11 * e^{-0,039 * Z_{t j}}\right)
$$

$E($ Index $)=$ Erreichbarkeitsindex

$G(Z j)=\quad$ jeweiliges Gewicht für das Ziel $(Z j)$

$f_{i j} \quad$ Widerstand laut Funktion zwischen dem

Quell- (i) und dem Zielort $(j)$

$Z_{t j}=\quad$ gemessene Reisezeit $(t)$ zum Zielort $(j)$

Der Erreichbarkeitsindex setzt sich aus zwei Termen zusammen: zum einen aus der jeweiligen Gewichtung, deren Anteil am Gesamtindex aus Abbildung 1 entnommen werden kann, und zum anderen aus der Reisezeit zur nächsten Einrichtung, die sich für die entsprechende Rasterzelle ergibt. Um nachvollziehen zu können, wie die Werte berechnet werden, wird an dieser Stelle ein Beispiel angeführt. Auf der Grundlage des Verkehrsmodells wird für die Zelle $\mathrm{x}$ eine Reisezeit von 20 Minuten zum nächsten Krankenhaus errechnet. Im zweiten Term wird für $Z_{\mathrm{tj}}$ der Wert 20 eingesetzt. Folgende Rechnung wird durchgeführt: $146,11 * \mathrm{e}^{-0,039 * 20}$. Daraus resultiert das Ergebnis 66,97. Dieser Wert wird mit dem ersten Term verrechnet, der als Gewicht für das nächste Krankenhaus einen Anteil von 0,03 vorsieht, was einem Wert von 2,0 entspricht. Im Sinne der Summenformel wird dieser Vorgang mit allen Zielen durchgeführt, die in Abbildung 1 aufgeführt sind, und letztlich ein Erreichbarkeitswert zwischen 0 und 100 ermittelt.

Für die Indexwerte des öffentlichen Verkehrs werden zudem die mittlere Bedienungs- und die Umstiegshäufigkeit an der jeweils nächsten Haltestelle von der entsprechenden Rasterzelle mit in den Indexwert einbezogen (vgl. Formel (2)). Die mittlere Bedienungshäufigkeit gibt an, wie oft die Haltestelle am Tag angefahren wird. Die Umstiegshäufigkeit zeigt, wie häufig im Durchschnitt umgestiegen werden muss. Die mittlere Bedienungshäufigkeit wurde aus Empfehlungswerten der Forschungsgesellschaft für Straßen- und Verkehrswesen abgeleitet, die für ländliche Räume einen 60-Minuten-Takt empfehlen, was in etwa einer Bedienungshäufigkeit von 20 Fahrten am Tag entspricht. Zusätzlich gelten maximal zwei Umstiege als zumutbar (FGSV 2010: $7 \mathrm{ff}$.). Dementsprechend wurde für die mittlere Bedienungshäufigkeit (MB) und mittlere Umstiegshäufigkeit (UH) folgende Formel ermittelt:

$$
\begin{aligned}
& E(\text { IndexÖV })=E(\text { Index }) \\
& +\left((M B-20) \times(2 \rightarrow[M B<20])^{*}\right) \\
& -\left(10 \rightarrow[U H>2]^{* *}\right)
\end{aligned}
$$

$E($ IndexÖV $)=$ Erreichbarkeitsindex für den ÖV $E($ Index $)=\quad$ Erreichbarkeitsindex (siehe Formel [1]) $M B=\quad$ mittlere Bedienhäufigkeit

$U H=\quad$ Umstiegshäufigkeit

* = $\quad$ der Faktor [2] wird nur dann berechnet, wenn der Wert für MB kleiner ist als 20

** $=\quad$ bei mehr als 2 Umstiegen werden pauschal 10 Punkte abgezogen

Genau wie in der Formel (2) werden die Werte für die einzelnen Ziele zur Berechnung des Gesamtindexes aufsummiert. Formel (2) liegt ein einfaches Prinzip zugrunde, das anhand des folgenden Beispiels erläutert wird: Im obe- 
ren Beispiel wird ein Wert von 66,67 Punkten für die Erreichbarkeit zum nächsten Krankenhaus ermittelt. Für die entsprechende Haltestelle in der Rasterzelle ist hinterlegt, wie oft diese pro Tag bedient wird; dies entspricht einer mittleren Bedienungshäufigkeit (MB) von 20. Liegt dieser Wert bei über 20, erhöht sich der Punktewert für die Haltestelle. Liegt dieser Wert bei unter 20, ergibt sich ein negativer Wert, die Punktzahl fällt dementsprechend niedriger aus. Da die Werte, die unter dem Schwellenwert der mittleren Bedienungshäufigkeit von 20 Punkten liegen, mit den Werten, die über dem Schwellenwert liegen, gleichgesetzt werden müssen, werden die negativen Werte verdoppelt (2 $\rightarrow[\mathrm{MB}<20])$. Liegt die mittlere Bedienungshäufigkeit beispielsweise bei 40, ergibt sich ein Indexwert von 86,67. Liegt die mittlere Bedienungshäufigkeit lediglich bei 10 , liegt der Wert bei 46,67. Der letzte Term, der die Umstiegshäufigkeit beinhaltet, führt zu einem Punkteabzug von 10 Punkten, wenn dieser höher als 2 liegt, das heißt, wenn eine Person für die Relationen an der entsprechenden Haltestelle im Tagesmittel mehr als zweimal umsteigen muss, um das entsprechende Ziel zu erreichen. Die Operation bezüglich der zweiten Formel wird für jede Einrichtung separat durchgeführt, bevor der Wert des Teilindizes endgültig in den Gesamtindex einbezogen wird.

Der Index fasst die Reisezeiten zu allen Standorten der Daseinsvorsorge zu einem Indexwert zusammen, um auf einen Blick zu verdeutlichen, in welchen Versorgungsbereichen die größte regionale Vulnerabilität besteht. Dabei werden die Kategorien der Einrichtungen unterschiedlich gewichtet, um eine realitätsnahe Relevanz bei der Grunddaseinsvorsorge und gewisse Wahlfreiheiten bei den einzelnen Einrichtungen abzubilden. So wird bei den Hausärzten, Lebensmittelgeschäften und Restaurants neben der nächsten auch die Erreichbarkeit zur übernächsten Einrichtung betrachtet. Bei den Fachärzten wird zusätzlich zwischen drei verschiedenen Zielen unterschieden. Die Bildung der Gewichtung des Erreichbarkeitsindexes erfolgt in mehreren Schritten, das Endergebnis ist in Abbildung 1 aufgeführt.

In einem ersten Schritt werden die empirisch abgeleiteten Weghäufigkeiten aus der Haushaltsbefragung in den Index integriert. Mit Ausnahme der Gesundheitsversorgung, spezieller Ziele der Nahversorgung und der Kindertagesstätten können für alle Ziele Gewichtungsanteile gemäß des in der Haushaltsbefragung aufgeführten Anteils der Wegzwecke abgeleitet werden. Ausbildungswege werden der Bildung, Einkaufswege des täglichen Bedarfs werden der Versorgung, Wege für private Erledigungen werden den Dienstleistungen und Behörden und Freizeitwege dem Bereich Freizeit und Kultur zugeordnet. Innerhalb der jeweiligen Bereiche werden die Gewichte für die Ziele gleich verteilt. Lediglich Supermärkte werden höher bewertet, da diesen eine Schlüsselrolle als Komplettversorger zukommt. Die Haushaltsbefragung umfasst auch weitere Wegzwecke wie Arbeitswege oder die Begleitung anderer Personen, die für die Verteilung der Gewichte aus der Grundgesamtheit ausgeklammert wurden.

Das Raum- und Verkehrsplanungstool wurde in Kooperation mit Vertretern aus den Gemeinden des Landkreises Calw sowie Vertretern des Landratsamts erarbeitet. Dazu fanden mehrere Workshops statt, in denen die spezifische Perspektive der Anwender in die Entwicklung des Werkzeugs eingeflossen ist. Es wurden zahlreiche Hinweise der Teilnehmer entgegengenommen und in den weiteren Projektaufbau übernommen. So konnten beispielsweise für den zweiten Schritt die Erkenntnisse in Bezug auf die Gewichtung des Gesundheitsbereichs angepasst werden. Die Wichtigkeit der hausärztlichen Versorgung wurde hier explizit betont und die Betrachtung von Frauen-, Kinder- und Zahnärzten als die relevanten Fachärzte hervorgehoben. Ziel des Workshops war es, wichtige Akteure im Landkreis in die Festlegung der Kennwerte der Erreichbarkeit sowie in die Entwicklung der Szenarien einzubinden.

In einem dritten Schritt werden theoretische Erkenntnisse integriert (z. B. BMVBS/BBSR 2011; Dinkel 2014; BMVI 2015b). Die Bereiche „Versorgung und Gesundheit“" werden dort als besonders wichtig erachtet und entsprechend aufgewertet. Im Gegenzug dazu fallen die Bereiche „Freizeit und Kultur“" sowie „Dienstleistungen und Behörden“" weniger ins Gewicht. Um den Index im Modell besser operationalisieren zu können, werden in einem vierten und letzten Schritt die Gewichte, wie nachfolgend aufgeführt, angepasst:

- Bildung: Da allen aufgeführten Bildungseinrichtungen der gleiche Stellenwert zukommt, werden die jeweiligen Zielkategorien gleich gewichtet.

- Gesundheit: Pflegeheime sind nur für eine bestimmte Bevölkerungsgruppe relevant, weshalb die Gewichtung zugunsten der Fachärzte reduziert wird.

- Nahversorgung: Lebensmittelgeschäften und Supermärkten kommt eine Schlüsselrolle in der Nahversorgung zu und sie sind dadurch noch stärker zu berücksichtigen.

- Freizeit und Kultur: In der Haushaltsbefragung wird ein hoher Anteil an Freizeitwegen gemessen (22,6\% aller Wege sind Freizeitwege). Zudem wurden einzelne Zielkategorien aufgewertet. So werden etwa Sportplätze bei BMVI (2015b) als eigene Kategorie bei der sozialen Infrastruktur betrachtet. Kirchen spielen eine wichtige Rolle für bestimmte Zielgruppen. Die wichtige soziale Funktion von Gaststätten stellte sich im Workshop mit den Bürgermeistern der Gemeinden des Landkreises heraus.

- Dienstleistungen und Behörden: Optiker und Jobcenter als Einrichtungen, die von der Gesamtbevölkerung vermeintlich eher sporadisch aufgesucht werden, erhalten je ein Gewicht weniger. 
Der Gesamtindex für die Qualität der regionalen Daseinsvorsorge setzt sich aus den Bereichen Bildung (15\%), Gesundheit (25\%), Versorgung (30\%), Freizeit und Kultur (20\%) sowie Dienstleistungen (10\%) zusammen. Der Index ist modular aufgebaut und kann je nach Fragestellung als Teilindex für einen Bereich oder auch für ein spezifisches Verkehrsmittel berechnet werden.

\subsection{Anwendung und Bewertung des Erreichbarkeitsindexes}

Mit dem Index bzw. den verschiedenen Indizes besteht die Möglichkeit, innerhalb der Gemeinden Gebiete zu identifizieren, die Defizite in der Erreichbarkeit von Zielen der Daseinsvorsorge aufweisen. Durch die Aggregation der Ziele kann zudem schnell herausgefunden werden, ob nur einzelne Bereiche betroffen sind oder ob für bestimmte Gebiete die gesamte soziale Infrastruktur defizitär ist. Hier zeigt sich der Mehrwert von Erreichbarkeitsanalysen, die mithilfe eines Indexes über die Betrachtung der Erreichbarkeit von Einzelzielen hinausgehen und somit eine integrierte Be- trachtung der Erreichbarkeitssicherung in ländlichen Räumen ermöglichen. Der Erreichbarkeitsindex ist somit ein Instrument, welches die Planung ganzheitlich unterstützen kann. Bei Darstellungen, die auf Reisezeiten beruhen, kann die Erreichbarkeit nur isoliert für eine Einrichtungskategorie betrachtet werden. Für Detailplanungen kann dies von großem Nutzen sein.

Die empirische Herleitung ist hinsichtlich der im vorangegangenen Abschnitt aufgeführten Genese konsistent. Die Wegedaten, die als Eingangsdaten für die Widerstandskurve dienen, können aus anderen bundesweiten Befragungen, wie „Mobilität in Deutschland“ (MID) oder dem „Deutschen Mobilitätspanel“ (MOP), abgeleitet werden und hätten somit eine größere annehmbare Grundgesamtheit. Im hier vorgestellten Ansatz wurde jedoch bewusst auf diese Datenquellen zugunsten der Haushaltsbefragung des Landkreises Calw verzichtet, um einen regionsspezifischen Index bilden zu können.

Das Grundprinzip der Skalierung von 0 bis 100 Punkten, abgeleitet von einer Widerstandsfunktion, folgt dem Prinzip des Walk Scores (2011) bei einer grundsätzlich veränder-

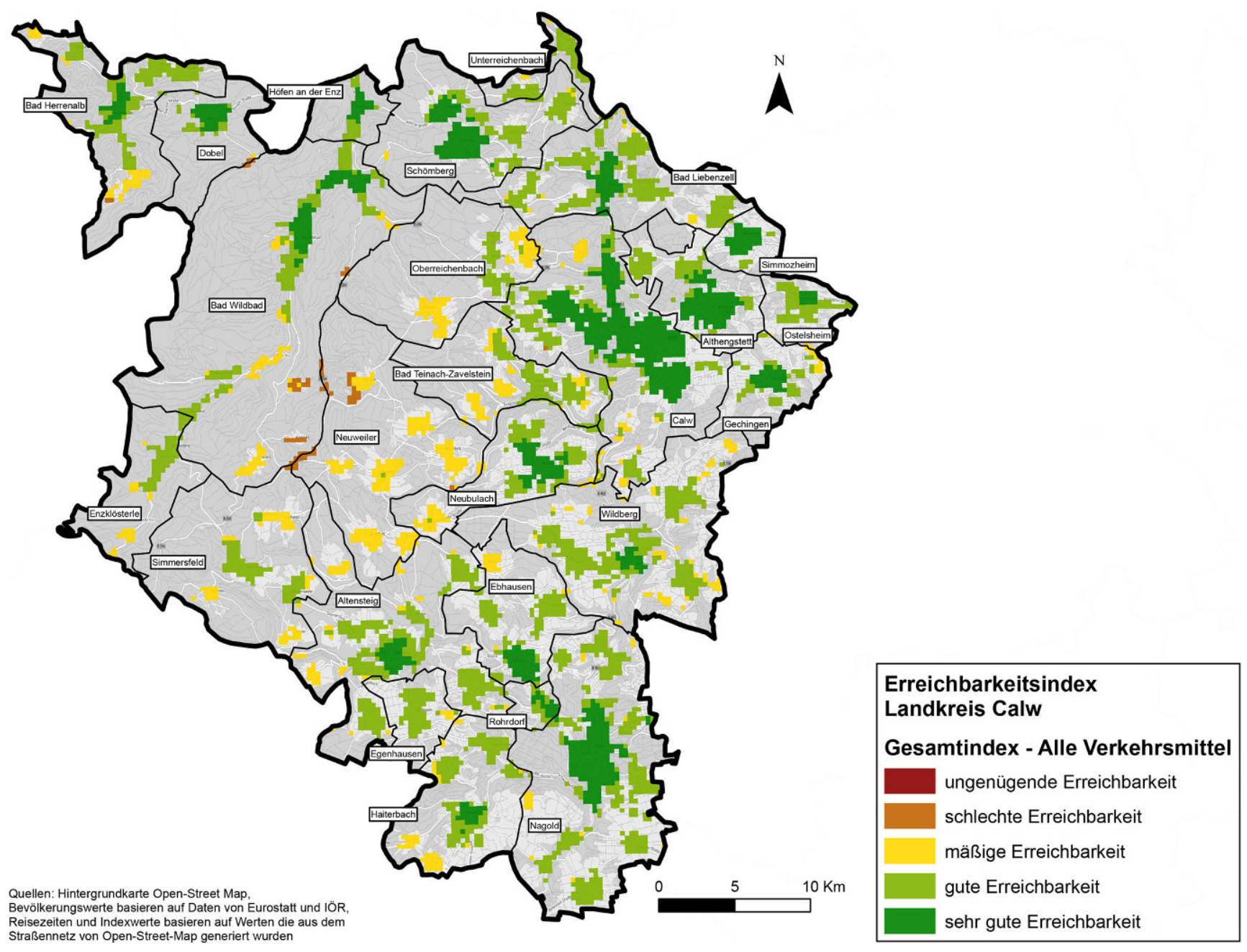

Abb. 5 Erreichbarkeitsindex für den Landkreis Calw (alle Verkehrsmittel) 


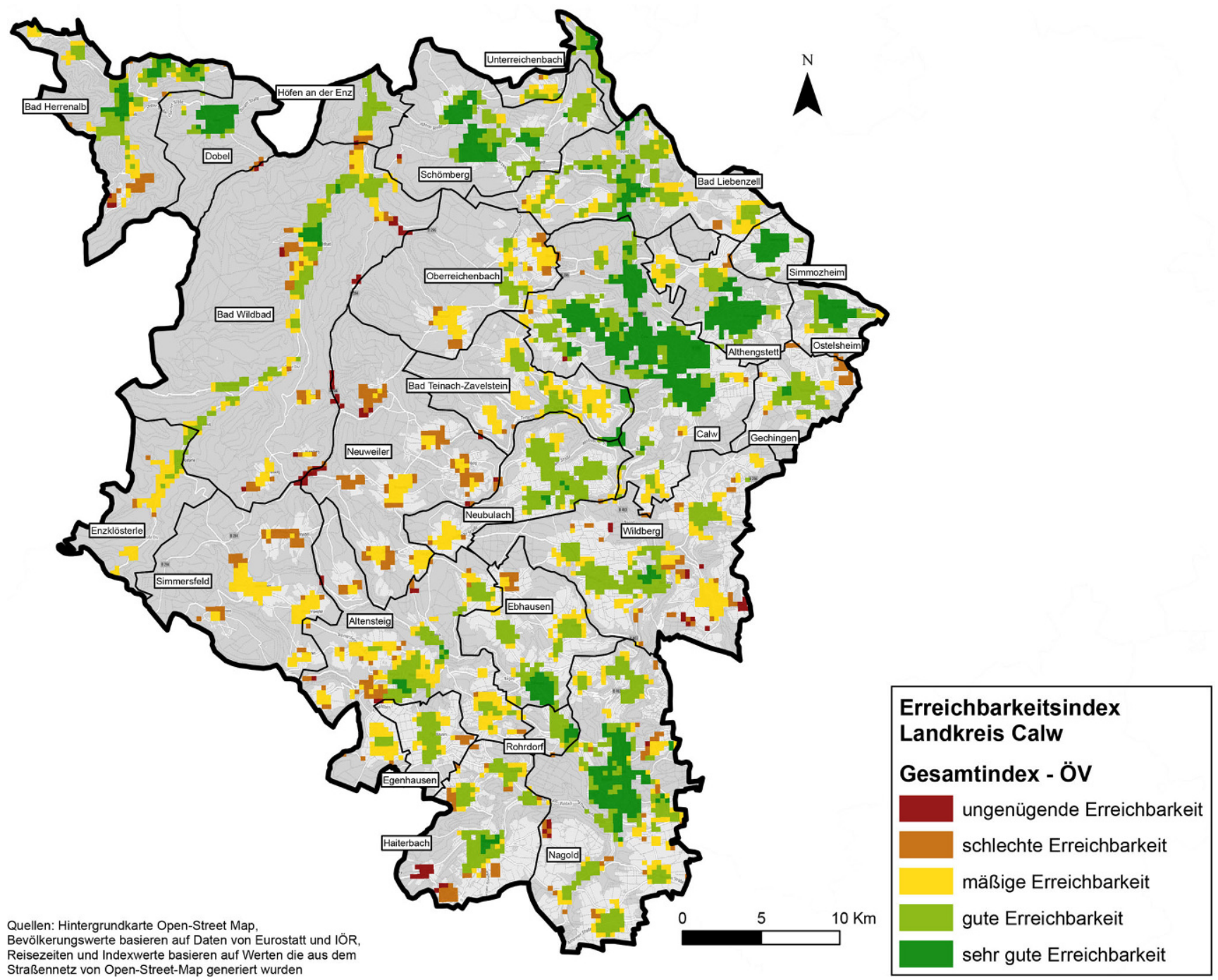

Abb. 6 Erreichbarkeitsindex für den öffentlichen Verkehr (ÖV)

ten Zielstruktur. Eine Neuerung in der Methodik stellt die Genese des Indexes dar. Hierfür werden sowohl quantitativ als auch qualitativ gewonnene mit theoretischen Erkenntnissen kombiniert, wodurch ein höheres Maß an Komplexität erreicht wird. Der Erklärungsgehalt ist gegenüber bisher angewandten Erreichbarkeitsindizes, wie dem Walk Score (2011) oder dem Index nach Dinkel (2014), höher, da alle Bereiche der Daseinsvorsorge in die Bildung des Indexes mit einbezogen werden. Die Komplexität erhöht jedoch für den Anwender die Anforderung an die Interpretation der Ergebnisse. Aufgrund dessen werden die Ergebnisse in leicht interpretierbare Karten übersetzt (vgl. Kapitel 5 und Abbildungen 5, 6, 7, 8 und 9).

Für die Planung erlaubt das Grundmodell die Klärung von Erreichbarkeitsfragen auf allen Ebenen und in einer Kleinräumigkeit, wie dies bis jetzt kaum ein Erreichbarkeitsmodell für einen gesamten Landkreis zulässt. Die Anwendung des Verkehrsplanungsprogramms VISUM und die
Nutzung von Verkehrszellen auf Rasterebene sind mit einem erhöhten Rechenaufwand verbunden. Somit ist das Instrument nicht ohne Hürden in jedem Landkreis implementierbar. Die Detailschärfe gestattet jedoch kleinräumige Planungen, ohne den Gesamtüberblick zu vernachlässigen, welcher über den Erreichbarkeitsindex ermöglicht wird.

\section{Indikatoren für regionale Planungsstrategien}

Für die kartographische Darstellung der kleinräumigen Modellierung wurden die Erreichbarkeitswerte, die zwischen 0 und 100 Punkten liegen, in Erreichbarkeitsqualitäten überführt. Bei Werten unter 20 Punkten wird die Erreichbarkeit als ungenügend betrachtet, bei unter 40 Punkten wird diese als schlecht und bei unter 60 Punkten als mäßig bewertet. 


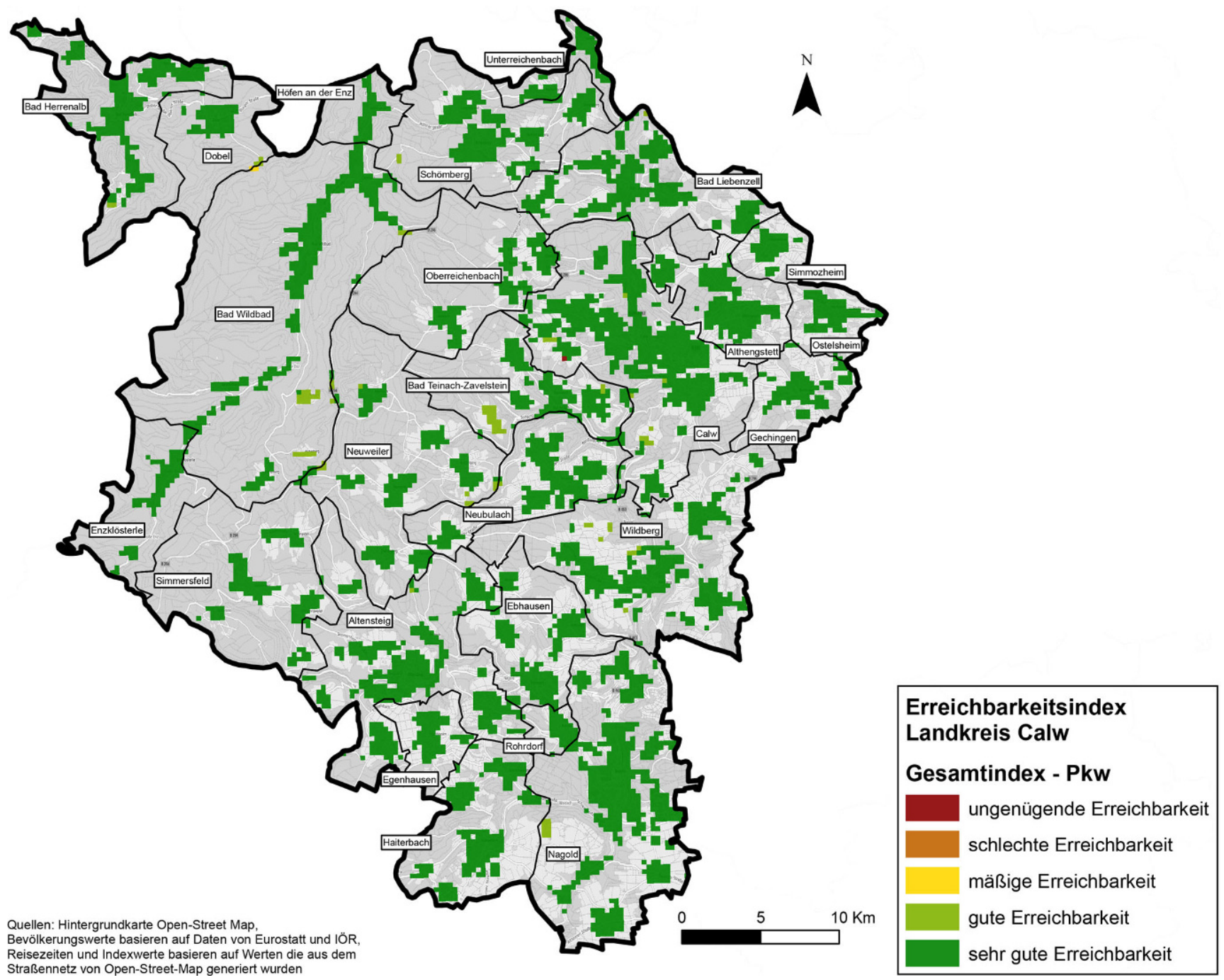

Abb. 7 Erreichbarkeitsindex für den Pkw

Bei Werten über 60 Punkten besteht eine gute und bei über 80 Punkten eine sehr gute Erreichbarkeit.

Abbildung 5 zeigt die Erreichbarkeitssituation gemittelt über alle Verkehrsmittel. Insgesamt sticht dort nur die Gemeinde Neuweiler hervor, die insgesamt keine gute Erreichbarkeit aufweist. In den restlichen Gemeinden besteht allenfalls in Randlagen eine nur mäßige Erreichbarkeit. Abbildung 6 zeichnet für den öffentlichen Verkehr ein anderes Bild. Der westliche Bereich des Landkreises Calw, der fernab der großen Kreisstädte und der Bahnlinie liegt, hat insgesamt eine mäßige bis schlechte Erreichbarkeit. Für die Gemeinden Neuweiler und Simmersfeld gilt dies sogar flächendeckend. Selbst in größeren Gemeinden wie Altensteig und Wildberg sowie in den Randbereichen der meisten weiteren Gemeinden des Landkreises ist die Erreichbarkeit zumindest teilweise unzureichend. Wer auf den öffentlichen Verkehr angewiesen ist, kann dort jegliche Ziele der Daseinsvorsorge nur eingeschränkt erreichen.
Die Erreichbarkeit mit dem Pkw ist flächendeckend sehr gut (vgl. Abbildung 7). Hier besteht kein Handlungsbedarf. Abbildung 8 führt die fußläufige Erreichbarkeit auf. In den zentralen Bereichen der größeren Gemeinden ist die Erreichbarkeit gut. In den ländlicheren Gebieten besteht, wie zu erwarten, keine Erreichbarkeit. Die Stadt Calw weist, bis auf die extremen Randlagen, beinahe flächendeckend eine gute Erreichbarkeit auf. Abbildung 8 verdeutlicht zudem die Konzentration der Einrichtungen in den zentralen Bereichen. Dort ist das Zufußgehen noch eine Alternative.

Da das Modell demographiesensibel ist, können nicht nur die räumliche Dimension abgebildet, sondern ebenso Gruppen identifiziert werden, die zukünftig eine veränderte oder schlechtere Erreichbarkeit vorfinden werden. So zeigt Abbildung 9 anhand des öffentlichen Verkehrs deutlich, dass einwohnerstarke Gemeinden weniger von schlechten Erreichbarkeitswerten betroffen sind als einwohnerschwache. Es wird deutlich, dass die Bereiche, in denen die Er- 


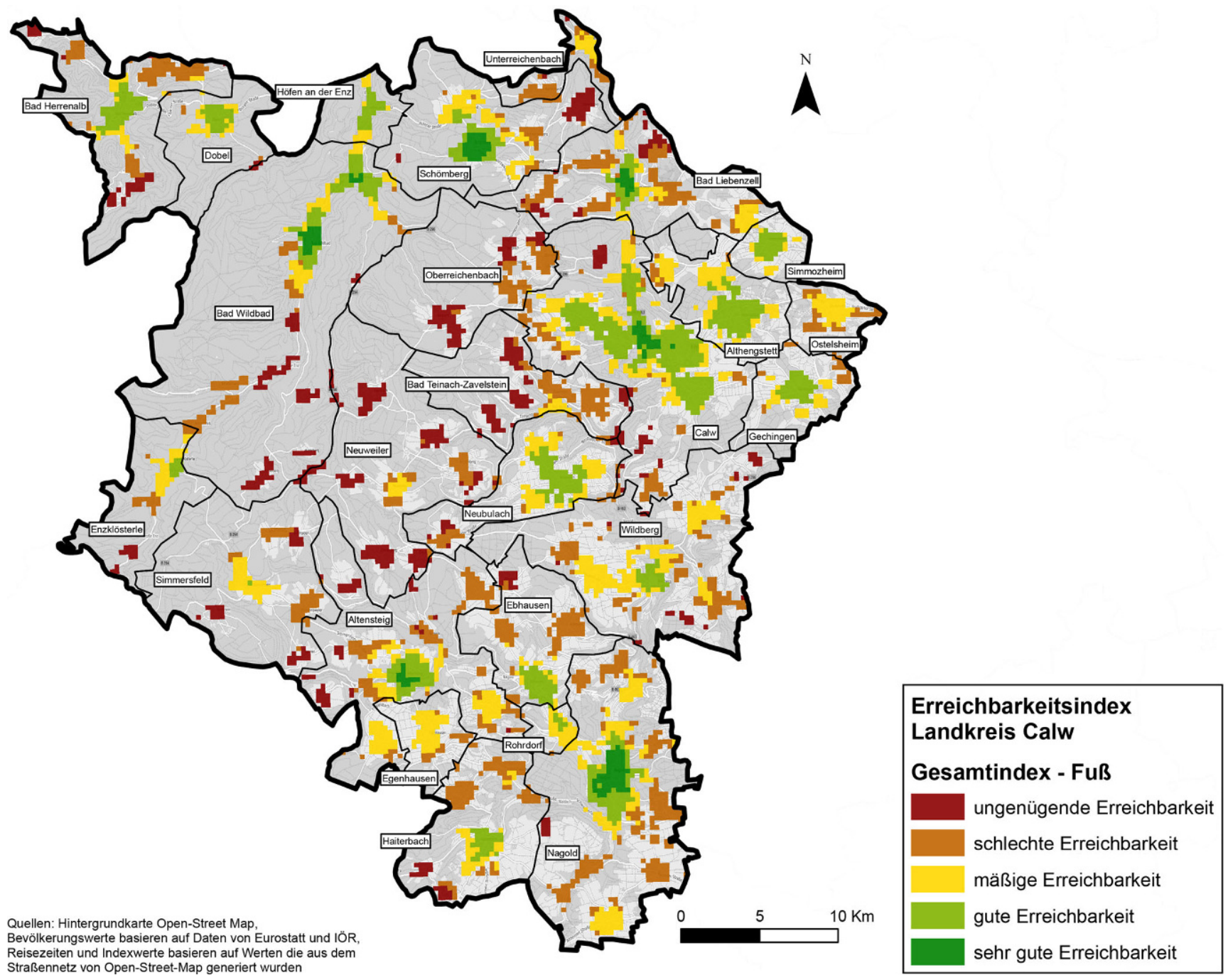

Abb. 8 Index der fußläufigen Erreichbarkeiten

reichbarkeit besonders schlecht ist, auch nur wenige Einwohner betreffen. Es wird jedoch auch sichtbar, dass insbesondere im zentralen ländlichen Bereich des Landkreises flächendeckende Mängel in der Erreichbarkeit vorherrschen.

Für die Praxis bedeutet dieses Ergebnis auf der Gesamtebene, dass schnell und übersichtlich die Bereiche herausgearbeitet sind, in denen eine wohnortnahe Daseinsvorsorge de facto nicht mehr oder kaum noch erreicht wird. In Szenarien besteht nun die Möglichkeit, Veränderungen bei den Standorten oder den Angebotsstrukturen vorzunehmen, um robuste Strategien zu entwickeln. Auf der Basis des sachlichen Ergebnisses können von regionalplanerischer Seite Kooperationen unter den betroffenen Gemeinden induziert werden. Diese können regionale Problemlösungen erarbeiten, wie z. B. eine Flächenbedienung des öffentlichen Verkehrs, die Organisation von Dienstleistungen, die zum Bürger kommen, oder die Umsetzung einer zentralen Gesund- heitseinrichtung, damit lange Wege zu verschiedenen Ärzten entfallen.

Der Kreis Calw weist insgesamt eine gute Erreichbarkeitssituation auf. Es sind jedoch flächendeckend Defizite in den dörflichen Gemeinden zu erkennen. Des Weiteren besteht für bestimmte Einrichtungsarten eine große Abhängigkeit zu den jeweils nächsten Zielen, wie dies am Vergleich der Abbildung 10 mit der Abbildung 11 anhand der hausärztlichen Versorgung deutlich wird. In diesem fiktiven Szenario fällt für jedes Bezugsraster die jeweils nächste Einrichtung weg. Der Vergleich mit der momentanen Erreichbarkeitssituation zeigt, wo die Bürger besonders vom jeweils nächsten Standort abhängig sind. So könnte in Orten wie Bad Teinach-Zavelstein, Altensteig oder Haiterbach, in denen die Erreichbarkeit flächendeckend zufriedenstellend ist, der Wegfall der nächsten Einrichtung die Versorgung erheblich verschlechtern. Interessanterweise liegen diese Ge- 


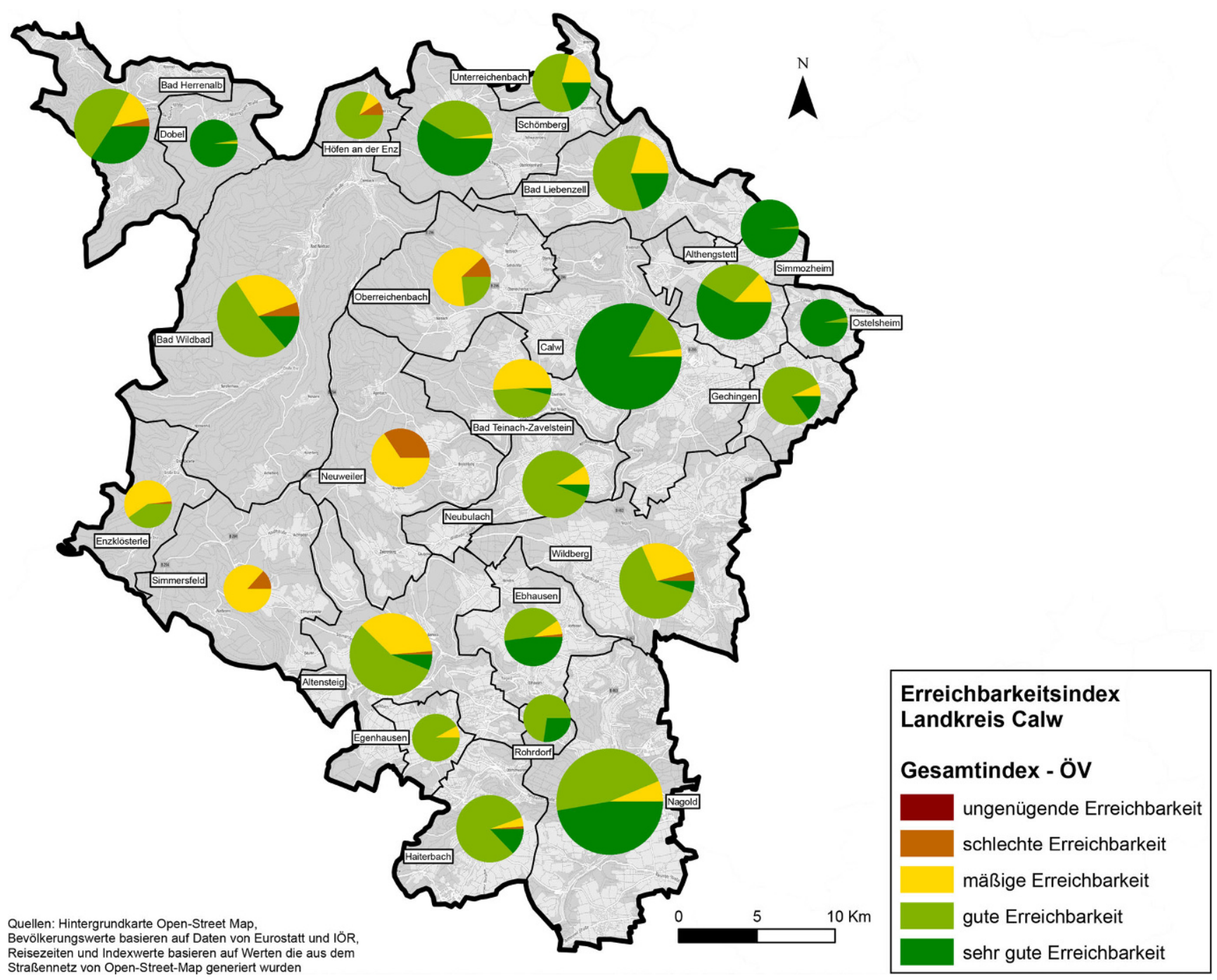

Abb. 9 Darstellung des Erreichbarkeitsindexes für den Öffentlichen Verkehr (ÖV) pro Einwohner in den jeweiligen Gemeinden

biete rund um die Gemeinde Neuweiler, der einzigen Gemeinde, in der sich keine Hausarztpraxis mehr befindet.

Eine Lösung könnte hier die Errichtung eines Ärztezentrums im zentralen Bereich des beschriebenen Risikogebietes darstellen. Der optimale Standort hierfür kann über das Modell ermittelt werden, da die Standorte modifizierbar sind. Da im betreffenden Gebiet die Anbindung an den öffentlichen Verkehr erwartungsgemäß schlecht ist, könnte hier verdeutlicht werden, wie ausgehend von einem potenziellen dezentralen Versorgungszentrum ein „Service to people“-Ansatz die ärztliche Versorgung in den dörflichen Gebieten des Landkreises erheblich verbessern könnte.

Das Beispiel demonstriert die Möglichkeiten, die das Instrument bietet. Es ist nicht statisch auf den Status quo ausgerichtet, sondern erlaubt durch Modifikation des Standort- oder Verkehrsmodells das Durchspielen unterschiedlicher Szenarien, die Lösungen aufzeigen, die sonst nicht gefunden werden könnten. Das Modell ermöglicht hierfür die planerische Grundlage mit der Darstellung von Erreichbarkeiten unterschiedlicher Versorgungsbereiche. Zudem lässt die Modellierung eine optimierte Standortplanung nach definierten Kriterien zu, wie Abbildung 12 exemplarisch darstellt. Auf der Basis der Erreichbarkeitswerte der Grundschulen wird deutlich, wie sich das Aufgeben von Standorten auf die Erreichbarkeit auswirkt und wo am besten Standorte im Hinblick auf die Erreichbarkeit reduziert werden können. Die Werte basieren auf jährlichen Personenstunden und stellen deren Reisezeitzuwachs pro Grundschüler dar. Anhand dieser Kartengrundlage kann der Landkreis Entscheidungen bei der Standortplanung treffen, deren Auswirkungen möglichst gering in Bezug auf die Erreichbarkeit und auf mögliche Umwelteffekte wären. Die beiden hier aufgeführten Szenarien zur hausärztlichen Versorgung und der Verteilung der Grundschulstandorte wurden im Rahmen der in Kapitel 4 angeführten Workshops gemeinsam mit Vertretern des Kreises entwickelt. 


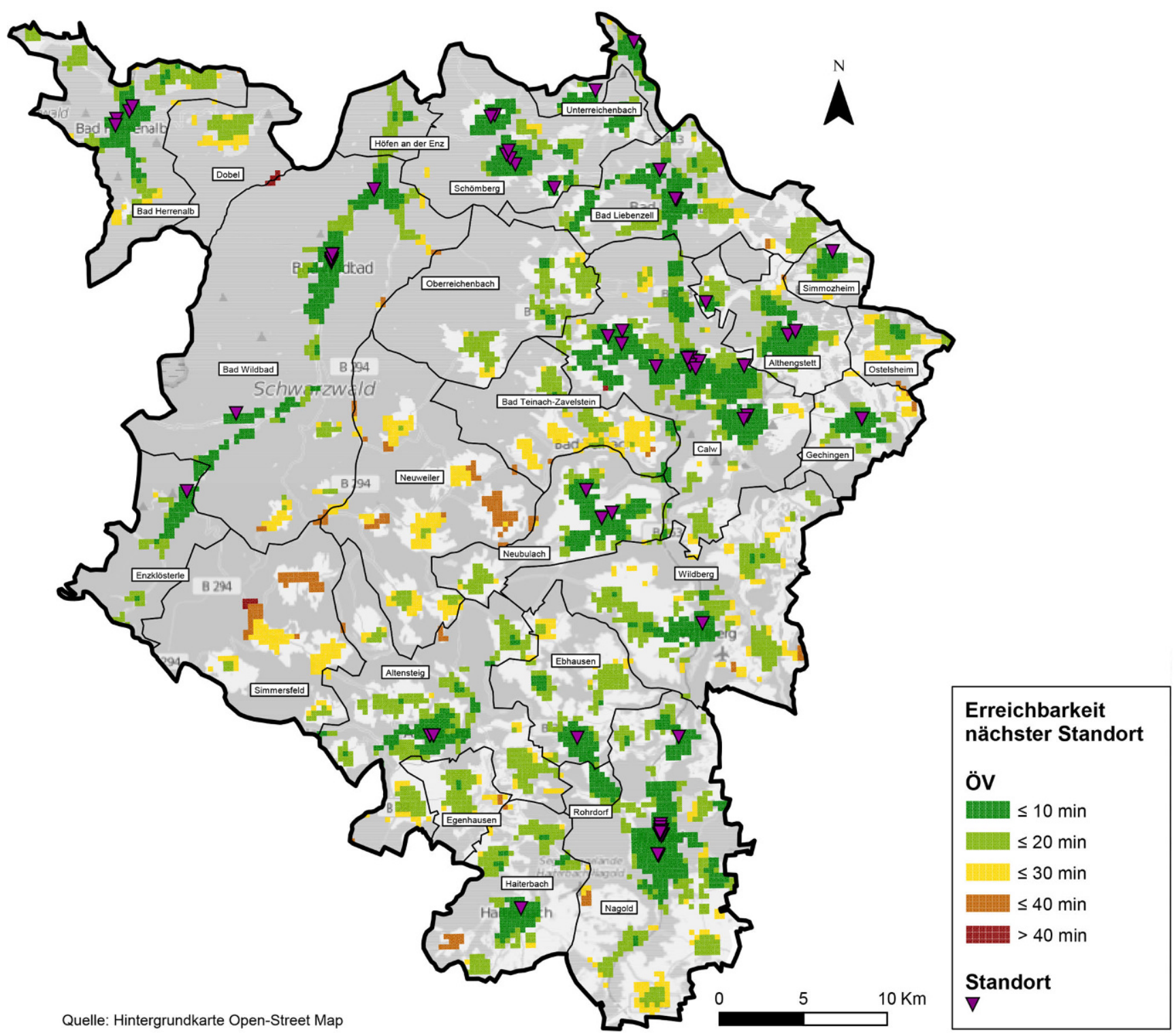

Abb. 10 Erreichbarkeit des nächsten Haus- und Allgemeinarztes mit dem öffentlichen Verkehr

Grundsätzlich sind die Parameter für die Standorte beliebig erweiterbar und auch das Angebot des öffentlichen Verkehrs kann im Modell modifiziert werden, um abschätzen zu können, welche Effekte die Veränderungsmaßnahmen des Verkehrsangebotes hätten. Durch die Dynamik unterscheidet sich das Modell von anderen Erreichbarkeitsmodellen dahingehend, dass es nicht nur den Stand der Erreichbarkeiten abbildet und defizitäre Bereiche aufzeigt, sondern auch die Wirkung potenzieller Maßnahmen darstellt und somit auf ihre Nachhaltigkeit hin überprüft. Eine Einschränkung besteht bei der Darstellung intermodaler Wegeketten, die im Modell nicht abgebildet werden können.

Das Modell bietet zudem die Möglichkeit, auf der Basis der Erreichbarkeitswerte Kosten-Nutzen-Rechnungen durchzuführen. So kann beispielsweise simuliert werden, welche Mengen an Kohlendioxid eingespart werden. Für Angebotserweiterungen im öffentlichen Verkehr können die Kosten pro Bürger errechnet werden. Die Ergebnisse solcher Szenarien können der Planung Aufschluss über die potenzielle Wirksamkeit von Maßnahmen geben und haben somit einen hohen Nutzen für die Erreichbarkeitsund Standortplanung.

Die Ergebnisse zeigen, dass mit dem Indikatorensystem ein Gesamtüberblick über alle Versorgungsbereiche zu generieren ist. Die Versorgungs- und Mobilitätsangebote im Landkreis Calw können gezielt verbessert und attraktiver gestaltet werden. Zudem haben Szenario-Funktionen das Potenzial, vulnerable Gebiete zu identifizieren und zukünftige Entwicklungen effizient zu planen. Das Modell liefert 


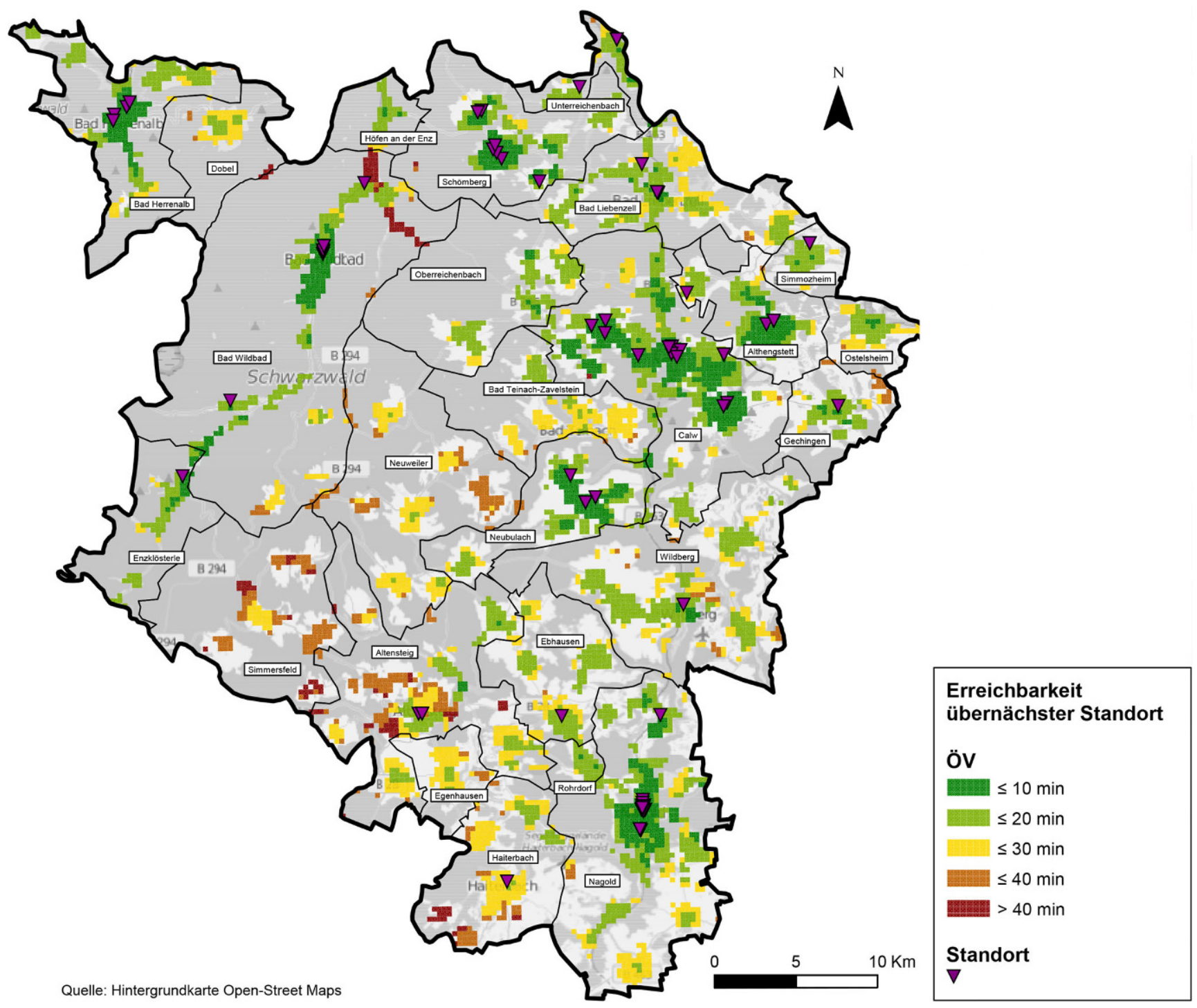

Abb. 11 Erreichbarkeit des übernächsten Haus- und Allgemeinarztes mit dem öffentlichen Verkehr

kleinräumige Ergebnisse, die für die Erreichbarkeitssicherung von zentraler Bedeutung sind.

\section{Anwendungsmöglichkeiten in der Fachplanung}

Die große Mehrheit, die individuell und mit dem Auto mobil ist, kennt in einem dicht besiedelten und mit einer hervorragenden Straßeninfrastruktur ausgestatteten Land kaum Erreichbarkeitsprobleme. Bevölkerungsgruppen, die ein weniger autoaffines Mobilitätsverhalten aufweisen bzw. keinen Zugang zu einem Auto haben, stehen oftmals vor großen Herausforderungen bei der Durchführung der Alltagsmobilität. Dennoch ist die Lebensqualität auf dem Land ein positiver Aspekt und die Installierung von Dorfläden, privaten Fahrgemeinschaften oder rollenden Lebensmit- telläden zeigt, dass neue Formen der Mobilität und neue Organisationsstrukturen die Attraktivität stärken können. Letztendlich wird die Attraktivität aber auch an Wahlmöglichkeiten gemessen.

Hier setzt das dargestellte Planungsinstrument an, da transparent mit objektiven Daten Defizite in der Erreichbarkeit von Standorten der Daseinsvorsorge für den Status quo sowie für zukünftige Bevölkerungs- und Angebotsveränderungen im Öffentlichen Personennahverkehr (ÖPNV) berechnet und visualisiert werden können. Für die Fachplanung bietet dies eine Reihe von Analysemöglichkeiten, um Handlungsempfehlungen zu untermauern und in einem geschützten und reproduzierbaren Rahmen Maßnahmen und Strategien auf ihre Wirkung zu überprüfen. Mit vergleichsweise einfachen Mitteln kann abgeschätzt werden, ob bestehende oder zukünftig befürchtete Erreichbarkeitsdefizite infolge von Standortschließungen durch Angebotsver- 


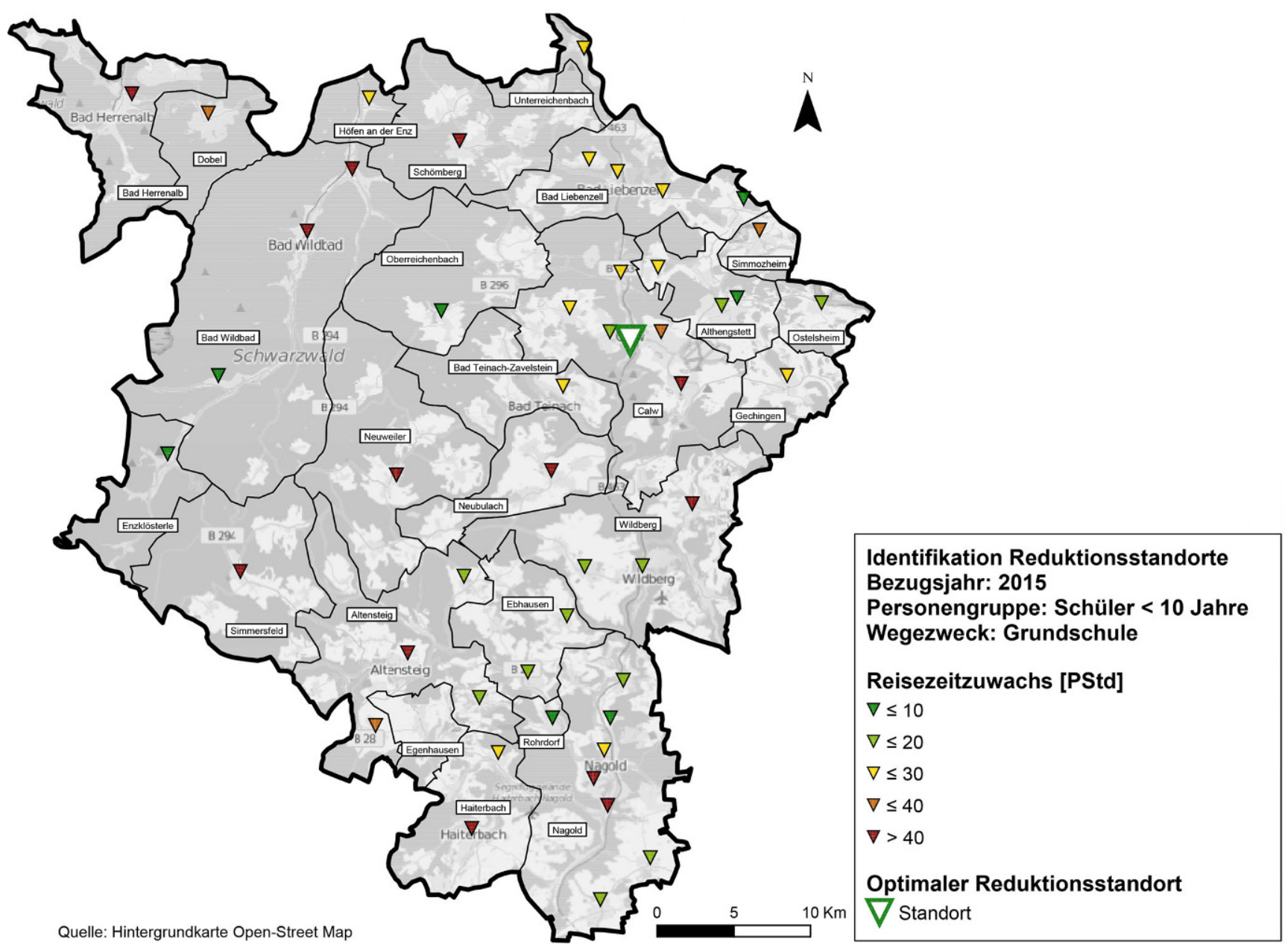

Abb. 12 Standortoptimierungspotenzial für Grundschulen

änderungen im öffentlichen Verkehr kompensiert und die Tragfähigkeit von Einrichtungen überprüft werden können. Darüber hinaus können Bevölkerungsgruppen identifiziert werden, die besonders durch Schließungen von Einrichtungen (wie z. B. Schließungen von Schulen oder Konzentration von Arztpraxen) oder einer Angebotsverschlechterung betroffen wären. Mit der Analyse können daher maßgeschneiderte Lösungen und Anpassungsstrategien entwickelt werden. Belegt wurde beispielsweise die Notwendigkeit eines Maßnahmenkataloges zur Sicherung der hausärztlichen Versorgung wegen der zunehmenden Überalterung der Ärzteschaft. Für den Masterplan „Hausärztliche Versorgung im Landkreis Calw" dienen die Erkenntnisse aus der Erreichbarkeitsstudie als tragfähige Grundlage.

Das Instrument lässt die Integration zusätzlicher Parameter und die Einbindung von unterschiedlichen Datenquellen $\mathrm{zu}$, um eine regionale Standortplanung auf der Grundlage aktueller und frei verfügbarer Daten zu ermöglichen. Da die Erreichbarkeitssituation in ländlichen Regionen mit Verkehrsmitteln des Umweltverbundes zunehmend angespannt sein wird, ist eine effektive Planung vor dem Hin- tergrund knapper kommunaler Finanzmittel unumgänglich. Ansonsten besteht die Gefahr, dass ökonomische und ökologische Tragfähigkeitsgrenzen unterschritten werden und Standorte der Daseinsvorsorge wegbrechen. Um solche Entwicklungen im Vorfeld quantitativ abschätzen zu können, liefert das vorgestellte Instrument einen wertvollen Beitrag. Die regionsspezifische Widerstandsfunktion in Kombination mit einer Zielgewichtung, die auf der Basis von empirischen und theoretischen Faktoren erarbeitet wurde, stellt methodisch eine Neuerung zur Bewertung von Erreichbarkeiten im Kontext der Daseinsvorsorge in ländlichen Räumen dar. Viele theoretische Überlegungen bisheriger Arbeiten wurden im Kontext der Modellierung übernommen (Frank/Sallis/Saelens et al. 2010; Walk Score 2011; Dinkel 2014). Die Wichtigkeit von Gaststätten als soziale Treffpunkte ist jedoch ein Beispiel für die regionsspezifische Anpassung, die bisher üblicherweise bei Darstellungen zur Erreichbarkeitssituation der Daseinsvorsorge keine Rolle spielten.

Die Ergebnisse zeigen, dass ein Werkzeug zur Begutachtung der Daseinsvorsorge offenlegen kann, wo aktuell 
Chancen und Defizite bestehen, um so den Impuls für eine interkommunale Zusammenarbeit zur Sicherung der Daseinsvorsorge zu liefern. Durch den modularen Aufbau des Planungswerkzeuges besteht die Möglichkeit der Übertragung auf andere ländliche Regionen. Das Prinzip der Kombination von Angebots- und Standortmodifikationen ermöglicht die Erstellung vielfältiger Szenarien zur Entwicklung der Erreichbarkeit. So können regionsspezifische Problemlagen herausgearbeitet werden, die aufzeigen, welche Konzepte überhaupt erforderlich sind. Zudem können nach definierten Optimierungskriterien (z. B. Minimierung der Reisezeit oder Emissionen) Vorschläge für neue Standorte oder Schließungen verortet und deren Effekte abgeschätzt werden.

Durch die offene Programmierung und einer kleinräumigen Abbildung der Infrastrukturen wird das Planungswerkzeug der Heterogenität ländlicher Räume gerecht. Dennoch muss auch kritisch reflektiert werden, dass ein ,Kümmerer in der Region oder in der Gemeinde notwendig ist, um auch eine Verstetigung im Blick zu haben. Bestimmte Kompetenzen - auch für die Interpretaion der Ergebnisse - sind zwingend notwendig. Welche Standort- und Mobilitätskonzepte die Regionen letztendlich für die Verbesserung der Erreichbarkeit anwenden, kann das Instrument nicht vorgeben. Hier sind die politischen Entscheidungsträger gefragt. $\mathrm{Ob}$ das entsprechende Umsetzungspotenzial in Form einer engagierten Bürgergesellschaft oder fachkundiger Verwaltungsangestellter vorhanden ist, muss jede Region selbst evaluieren. Die Erreichbarkeitssicherung sollte jedoch mit der sachlichen Analyse der Problemlage beginnen, erst dann sollte die Konzeptentwicklung folgen.

\section{Literatur}

ARL - Akademie für Raumforschung und Landesplanung (2016): Daseinsvorsorge und gleichwertige Lebensverhältnisse neu denken. Perspektiven und Handlungsfelder. Hannover. = Positionspapier aus der ARL 108.

BBSR - Bundesinstitut für Bau-, Stadt- und Raumforschung (Hrsg.) (2017): Raumordnungsbericht 2017. Daseinsvorsorge sichern. Bonn.

Bertolini, L.; Le Clercq, F.; Capoen, L. (2005): Sustainable accessibility: a conceptual framework to integrate transport and land use plan-making. Two test-applications in the Netherlands and a reflection on the way forward. In: Transport Policy 12, 3, 207-220. https://doi.org/10.1016/j.tranpol.2005.01.006

BMVBS - Bundesministerium für Verkehr, Bau und Stadtentwicklung; BBSR - Bundesinstitut für Bau-, Stadt-, und Raumforschung (Hrsg.) (2009): Handbuch zur Planung flexibler Bedienungsformen im ÖPNV. Bonn.

BMVBS - Bundesministerium für Verkehr, Bau und Stadtentwicklung; BBSR - Bundesinstitut für Bau-, Stadt- und Raumforschung (Hrsg.) (2011): Regionalstrategie Daseinsvorsorge - Denkanstöße für die Praxis. Berlin.

BMVI - Bundesministerium für Verkehr und digitale Infrastruktur (Hrsg.) (2015a): Anpassungsstrategien zur regionalen Daseinsvorsorge. Berlin $=$ MORO Praxis 2/2015.
BMVI - Bundesministerium für Verkehr und digitale Infrastruktur (Hrsg.) (2015b): Kennzahlen in der Daseinsvorsorge. Berlin. = BMVI-Online-Publikation 01/2015.

BMVI - Bundesministerium für Verkehr und digitale Infrastruktur (Hrsg.) (2016): Integrierte Mobilitätskonzepte zur Einbindung unterschiedlicher Mobilitätsformen in ländlichen Räumen. Berlin. = BMVI-Online-Publikation 04/2016.

Canzler, W.; Karl, A. (2010): Mit der Subjektförderung zur Mobilitätssicherung? Chancen und Barrieren für einen innovativen Landverkehr. In: Informationen zur Raumentwicklung 7, 505-515.

Dinkel, M. (2014): Der Einfluss der Nahmobilität auf Immobilienpreise in urbanen Räumen. Dissertation, Technische Universität Kaiserslautern.

FGSV - Forschungsgesellschaft für Straßen- und Verkehrswesen (2006): Hinweise zu verkehrlichen Konsequenzen des demografischen Wandels. Köln. = FGSV 144.

FGSV - Forschungsgesellschaft für Straßen- und Verkehrswesen (2010): Empfehlungen für Planung und Betrieb des öffentlichen Personennahverkehrs. Forschungsprojekt des Forschungsprogramms Stadtverkehr (FoPS) FA-Nr. 70.837/2009 im Auftrag des Bundesministeriums für Verkehr, Bau und Stadtentwicklung. Köln.

Frank, L. D.; Sallis, J. F.; Saelens, B. E.; Leary, L.; Cain, K.; Conway, T. L.; Hess, P. M. (2010): The development of a walkability index: application to the Neighborhood Quality of Life Study. In: British Journal of Sports Medicine 44, 13, 924-933. https://doi.org/10. 1136/bjsm.2009.058701

Friedrich, M.; Siedentop, S.; Ahlmeyer, F.; Wittowsky, D.; Rönsch, S.; Hartl, M.; Petzold, M. (2016): Erreichbarkeitssicherung im ländlichen Raum - Entwicklung und Erprobung eines Werkzeugs zur Bewertung der Wirkungen von Standortentwicklungen der sozialen Infrastruktur und Angebotsveränderungen des IV/ÖV (Abschlussbericht). Stuttgart/Dortmund.

Gather, M.; Kagermeier, A.; Lanzendorf, M. (2008): Geographische Mobilitäts- und Verkehrsforschung. Berlin/Stuttgart.

Geurs, K. T.; van Wee, B. (2004): Accessibility evaluation of landuse and transport strategies: review and research directions. In: Journal of Transport Geography 12, 2, 127-140. https://doi.org/ 10.1016/j.jtrangeo.2003.10.005

Gölz, H. (2007): Aufbau eines Verkehrsmodells für die Radverkehrsplanung in Stuttgart. Diplomarbeit, Lehrstuhl Verkehrsplanung und Verkehrsleittechnik, Universität Stuttgart.

Halden, D. (2002): Using accessibility measures to integrate land use and transport policy in Edinburgh and the Lothians. In: Transport Policy 9, 4, 313-324. https://doi.org/10.1016/S0967070X(02)00017-3

Kemper, F-J. (2004): Regionale Bevölkerungsentwicklung zwischen Wachstum und Schrumpfung. In: Geographische Rundschau 56, 9, 20-25.

Kickner, S. (1998): Kognition, Einstellung und Verhalten - Eine Untersuchung des individuellen Verkehrsverhaltens in Karlsruhe. Karlsruhe. $=$ Schriftenreihe des Instituts für Verkehrswesen, Universität Karlsruhe (TH) 56.

Kilper H.; Müller, B. (2005): Demographischer Wandel in Deutschland. In: Geographische Rundschau 57, 3, 36-41.

Neumeier, S. (2013): Modellierung der Erreichbarkeit öffentlicher Apotheken. Untersuchung zum regionalen Versorgungsgrad mit Dienstleistungen der Grundversorgung. Braunschweig. = Thünen Working Paper 14.

Neumeier, S. (2014): Modellierung der Erreichbarkeit von Supermärkten und Discountern. Untersuchung zum regionalen Versorgungsgrad mit Dienstleistungen der Grundversorgung. Braunschweig. $=$ Thünen Working Paper 16 .

Neumeier, S. (2017): Haus- und Facharzterreichbarkeit in Deutschland. Regionalisierte Betrachtung auf Basis einer GIS-Erreichbarkeitsanalyse. In: Raumplanung 192, 4, 30 -37.

Ottmann, P. (2010): Abbildung demographischer Prozesse in Verkehrsentstehungsmodellen mit Hilfe von Längsschnittdaten. Karlsruhe. 
= Schriftenreihe des Instituts für Verkehrswesen, Karlsruher Institut für Technologie 69.

Proske, M. (2011): Demographischer Wandel und Daseinsvorsorge. Auswirkungen kommunaler Angebote auf die Wohnortwahl. Dissertation, Technische Universität Kaiserslautern.

Rauch, S.; Rauh, J. (2016): Verfahren der GIS-Modellierung von Erreichbarkeiten für Schlaganfallversorgungszentren. In: Raumforschung und Raumordnung 74, 5, 437-450. https://doi.org/10. 1007/s13147-016-0432-5

Rode, P.; Floater, G. (2014): Accessibility in Cities: Transport and Urban Form. London. = NCE Cities Paper 03.

Schwarze, B. (2005): Erreichbarkeitsindikatoren in der Nahverkehrsplanung. Dortmund. = Arbeitspapier 184 des Instituts für Raumplanung der Technischen Universität Dortmund.
Walk Score (2011): Walk Score Methodology. Seattle.

Wittowsky, D. (2008): Dynamische Informationsdienste im ÖPNV Nutzerakzeptanz und Modellierung. Karlsruhe. = Schriftenreihe des Instituts für Verkehrswesen, Karlsruher Institut für Technologie 68 .

Wulfhorst, G.; Büttner, B.; Ji, C. (2017): The TUM Accessibility Atlas as a tool for supporting policies of sustainable mobility in metropolitan regions. In: Transportation Research Part A: Policy and Practice 104, 121-136. https://doi.org/10.1016/j.tra.2017.04.012

Yigitcanlar, T.; Sipe, N.; Evans, R.; Pitot, M. (2007): A GIS-based land use and public transport accessibility indexing model. In: Australian Planner 44, 3, 30-37. https://doi.org/10.1080/07293682.2007. 9982586 